\title{
Symmetry group analysis of weak solutions
}

\author{
N. Đapić $\quad$ M. Kunzinger S. Pilipović
}

\begin{abstract}
Methods of Lie group analysis of differential equations are extended to weak solutions of (linear and nonlinear) PDEs, where the term "weak solution" comprises the following settings:

(a) Distributional solutions.

(b) Solutions in generalized function algebras.

(c) Solutions in the sense of association (corresponding to a number of weak or integral solution concepts in classical analysis).

Factorization properties and infinitesimal criteria are developed that allow to treat all three settings simultaneously, thereby unifying and extending previous work in this area.
\end{abstract}

Key words. Algebras of generalized functions, Lie symmetries of differential equations, group analysis, Colombeau algebras.

Mathematics Subject Classification (2000). 46F30, 22E70, 35Dxx, 35A30.

\section{Introduction}

Local symmetries for equations with weak type solutions, such as, e.g., conservation laws, involve different constraints depending on the framework in which the equations are analyzed. The aim of this paper is to study symmetry properties of differential equations involving singular (in particular: distributional) terms through an analysis of symmetries in distribution spaces and generalized function algebras, as well as associated (i.e., weak type) symmetries. A main ingredient in our analysis will be the determination of infinitesimal criteria for these solution concepts.

Investigations in this direction have been initiated by Methé ([25), Tengstrand (34]), Szmydt and Ziemian ( 31, 32, 33]) and have been systematically pursued by Berest and Ibragimov ([3, 4, 5, 6, 15, 16]) in the distributional setting. More recently, in [19, 20, 21], an extension of the purely distributional methods applied so far has been given that allows to also consider nonlinear equations involving singularities. The basic tool allowing for such an extension is Colombeau's

\footnotetext{
Institute of Mathematics, University of Novi Sad, Trg D. Obradovića 4, 21000 Novi Sad, Yugoslavia. E-mail: nenad@unsim.ns.ac.yu

Institut für Mathematik, Universität Wien, Strudlhofg. 4, A-1090 Wien, Austria. E-mail: Michael.Kunzinger@univie.ac.at

Institute of Mathematics, University of Novi Sad, Trg D. Obradovića 4, 21000 Novi Sad, Yugoslavia. E-mail: pilipovic@unsim.ns.ac.yu
} 
theory of algebras of generalized functions. In what follows, on the one hand we are going to continue the analysis of [21], and on the other hand we shall establish connections between the distributional criteria developed in [4, 5 , and the Colombeau-type methods given in 21]. Moreover, the framework of generalized function algebras enables us to study symmetry properties of associated solutions (i.e. of weak or integral solutions) by the same methods.

To begin with, let us fix some notations concerning group analysis of differential equations and Colombeau's theory of generalized functions. Our principal reference for symmetries of differential equations is [28, whose terminology we shall follow closely. Let $\mathcal{M}$ be an open subset of $\mathbb{R}^{p+q}$ (in what follows, $p$ will be the number of independent variables of a system of differential equations, $q$ the number of dependent variables) and $G$ a Lie group acting regularly on $\mathcal{M}$. For $x \in \mathbb{R}^{p}, u \in \mathbb{R}^{q}$ and $(x, u) \in \mathcal{M}$ we write

$$
(\tilde{x}, \tilde{u})=g \cdot(x, u)=\left(\Xi_{g}(x, u), \Phi_{g}(x, u)\right)
$$

If $\Xi_{g}(x, u)=\Xi_{g}(x), g \in G$, then $G$ is called projectable. Elements of the Lie algebra of $G$ as well as the corresponding vector fields on $\mathcal{M}$ will typically be denoted by $\mathbf{v}$.

Put $\mathcal{X}=\mathbb{R}^{p}, \mathcal{U}=\mathbb{R}^{q}$. By identifying a function $f: \Omega \subset \mathcal{X} \rightarrow \mathcal{U}$ with its graph $\Gamma_{f}=\{(x, f(x)) \mid x \in \Omega\} \subset \mathcal{X} \times \mathcal{U}$, the action of $g \in G$ onto $f$ is defined (locally) by

$$
g \cdot f=\tilde{f}=\left(\Phi_{g} \circ\left(\operatorname{id}_{\mathcal{X}} \times f\right)\right) \circ\left(\Xi_{g} \circ\left(\operatorname{id}_{\mathcal{X}} \times f\right)\right)^{-1}
$$

where $\operatorname{id}_{\mathcal{X}}$ is the identity mapping on $\mathcal{X}$; in the projectable case this specializes to

$$
g \cdot f=\Phi_{g} \circ\left(\operatorname{id}_{\mathcal{X}} \times f\right) \circ \Xi_{g}^{-1} .
$$

Set $\mathcal{U}^{(n)}=\mathcal{U} \times \mathcal{U}_{1} \times \ldots \times \mathcal{U}_{n}, \mathcal{M}^{(n)}=\mathcal{M} \times \mathcal{U}_{1} \times \ldots \times \mathcal{U}_{n}$. Here $\mathcal{U}_{k}=\mathbb{R}^{q p_{k}}$, with coordinates $u_{J}^{\alpha}, \alpha=1, \ldots, q, J=\left(j_{1}, \ldots, j_{k}\right) \in\{1, \ldots, p\}^{k}$ such that $j_{1} \leq j_{2} \leq$ $\ldots \leq j_{k}$ and $p_{k}=\left(\begin{array}{c}p+k-1 \\ k\end{array}\right)$, is the number of different partial derivatives of order $k$ of a scalar valued smooth function of $p$ variables. Elements of $\mathcal{U}^{(n)}$ are denoted by $u^{(n)} . \mathcal{M}^{(n)}$ is called the $n$-jet space of $\mathcal{M}$ and we set $N:=\operatorname{dim}\left(\mathcal{M}^{(n)}\right)$. The coordinates on $\mathcal{M}^{(n)}$ will also be written as $\left(z_{1}, \ldots, z_{p}, z_{p+1}, \ldots, z_{N}\right)=\left(x, u^{(n)}\right)$. For any $f: \Omega \subseteq \mathcal{X} \rightarrow \mathbb{R}^{q}$, the $n$-th prolongation or $n$-jet of $f$ is the function $\operatorname{pr}^{(n)} f: \Omega \rightarrow \mathcal{U}^{(n)}$ formed by $f$ and its derivatives up to order $n$. The $n$-th prolongation of a group action $g$ or vector field $\mathbf{v}$ is written as $\operatorname{pr}^{(n)} g$ or $\operatorname{pr}^{(n)} \mathbf{v}$, respectively.

Let $\Sigma$ be a system of differential equations with $p$ variables and $q$ unknown functions of the form

$$
\Delta_{i}\left(x, u^{(n)}\right)=0, \quad i=1, \ldots, s
$$

(with $\Delta_{i}$ smooth for all $i$ ). We shall henceforth assume that (11) is nondegenerate (i.e. locally solvable and of maximal rank, see 28]). Any $f: \Omega \subset \mathcal{X} \rightarrow \mathcal{U}$ which solves the system on its domain will be called a solution. This amounts to saying that the graph of the $n$-jet of $f, \Gamma_{f}^{(n)}$ is contained in the zero-set $\Sigma_{\Delta}$ of $\Delta$. A symmetry group of $\Sigma$ is a local transformation group on $\mathcal{X} \times \mathcal{U}$ such that if $f$ is a solution of the system, $g \in G$ and $g \cdot f$ is defined then also $g \cdot f$ is a solution of $\Sigma$. 
Next, let us shortly recall some basic definitions from Colombeau's theory of generalized functions ([1], [7], [9], [10], [11], [27]). For notational simplicity we are going to work in the so-called "special" Colombeau algebra $\mathcal{G}(\Omega)$, defined as the quotient algebra $\mathcal{E}_{M}(\Omega) / \mathcal{N}(\Omega)$, where

$$
\begin{aligned}
\mathcal{E}_{M}(\Omega):= & \left\{\left(u_{\varepsilon}\right)_{\varepsilon \in I} \in \mathcal{C}^{\infty}(\Omega)^{I}: \forall K \subset \subset \Omega, \forall \alpha \in \mathbb{N}_{o}^{n} \exists p \in \mathbb{N}\right. \text { with } \\
& \left.\sup _{x \in K}\left|\partial^{\alpha} u_{\varepsilon}(x)\right|=O\left(\varepsilon^{-p}\right) \text { as } \varepsilon \rightarrow 0\right\} \\
\mathcal{N}(\Omega):= & \left\{\left(u_{\varepsilon}\right)_{\varepsilon \in I} \in \mathcal{C}^{\infty}(\Omega)^{I}: \forall K \subset \subset \Omega, \forall \alpha \in \mathbb{N}_{o}^{n} \forall q \in \mathbb{N}\right. \\
& \left.\sup _{x \in K}\left|\partial^{\alpha} u_{\varepsilon}(x)\right|=O\left(\varepsilon^{q}\right) \text { as } \varepsilon \rightarrow 0\right\}
\end{aligned}
$$

Here $I=(0,1] \cdot \mathcal{G}(\Omega)$ is a differential algebra (with componentwise operations) and $\Omega \rightarrow \mathcal{G}(\Omega)$ is a fine sheaf on $\mathbb{R}^{n}$. The equivalence class of $\left(u_{\varepsilon}\right)_{\varepsilon \in I}$ in $\mathcal{G}(\Omega)$ will be denoted by $U=\operatorname{cl}\left[\left(u_{\varepsilon}\right)_{\varepsilon \in I}\right]$ or $\operatorname{cl}\left[u_{\varepsilon}\right]$ for short.

We shall make use of the function spaces

$$
\begin{aligned}
\mathcal{D}(\Omega) & =\left\{f \in \mathcal{C}^{\infty}(\Omega): \operatorname{supp}(f) \text { compact }\right\} \\
\mathcal{S}\left(\mathbb{R}^{n}\right) & =\left\{f \in \mathcal{C}^{\infty}\left(\mathbb{R}^{n}\right): \forall q>0 \forall \alpha \in \mathbb{N}_{0}^{n} \sup _{x \in \mathbb{R}^{n}}(1+|x|)^{q}\left|\partial^{\alpha} f(x)\right|<\infty\right\} \\
\mathcal{O}_{M}(\Omega) & =\left\{f \in \mathcal{C}^{\infty}(\Omega): \forall \alpha \in \mathbb{N}_{0}^{n} \exists p>0 \sup _{x \in \Omega}(1+|x|)^{-p}\left|\partial^{\alpha} f(x)\right|<\infty\right\}
\end{aligned}
$$

$\mathcal{D}(\Omega)$ is the space of test functions on $\Omega$, elements of $\mathcal{S}\left(\mathbb{R}^{n}\right)$ and $\mathcal{O}_{M}\left(\mathbb{R}^{n}\right)$ are called rapidly decreasing and slowly increasing, respectively.

The algebra $\mathcal{G}_{\tau}(\Omega)=\mathcal{E}_{\tau}(\Omega) / \mathcal{N}_{\tau}(\Omega)$ of tempered generalized functions is defined by

$$
\begin{aligned}
& \mathcal{E}_{\tau}(\Omega)=\left\{\left(u_{\varepsilon}\right)_{\varepsilon \in I} \in\left(\mathcal{O}_{M}(\Omega)\right)^{I}: \forall \alpha \in \mathbb{N}_{o}^{n} \exists p>0\right. \\
&\left.\sup _{x \in \Omega}(1+|x|)^{-p}\left|\partial^{\alpha} u_{\varepsilon}(x)\right|=O\left(\varepsilon^{-p}\right)(\varepsilon \rightarrow 0)\right\} \\
& \mathcal{N}_{\tau}(\Omega)=\left\{\left(u_{\varepsilon}\right)_{\varepsilon \in I} \in\left(\mathcal{O}_{M}(\Omega)\right)^{I}: \forall \alpha \in \mathbb{N}_{o}^{n} \exists p>0 \forall q>0\right. \\
&\left.\sup _{x \in \Omega}(1+|x|)^{-p}\left|\partial^{\alpha} u_{\varepsilon}(x)\right|=O\left(\varepsilon^{q}\right)(\varepsilon \rightarrow 0)\right\}
\end{aligned}
$$

By componentwise insertion, elements of $\mathcal{G}(\Omega)$ and $\mathcal{G}_{\tau}(\Omega)$ can be composed with slowly increasing functions. Next, choose $\rho \in \mathcal{S}\left(\mathbb{R}^{n}\right)$ such that $\int \rho(x) d x=1$ and $\int \rho(x) x^{\alpha} d x=0$ for all $\alpha \in \mathbb{N}_{0}^{n}$ with $|\alpha| \geq 1$. Then $\mathcal{E}^{\prime}(\Omega)$ (the space of compactly supported distributions) is linearly embedded into $\mathcal{G}(\Omega)$ via $\iota: u \rightarrow \operatorname{cl}\left[\left(u * \rho_{\varepsilon}\right)_{\varepsilon \in I}\right]$ (where $\rho_{\varepsilon}=\frac{1}{\varepsilon^{n}} \rho(\dot{\bar{\varepsilon}})$ ). Moreover $\iota$ coincides with the identical embedding $\sigma: f \rightarrow$ $\operatorname{cl}\left[(f)_{\varepsilon \in I}\right]$ on $\mathcal{D}(\Omega)$, so $\mathcal{D}(\Omega)$ becomes a subalgebra of $\mathcal{G}(\Omega)$ via $\iota$. Finally, there is a unique sheaf morphism $\hat{\imath}$ extending $\iota$ to $\mathcal{C}^{\infty}(.) \hookrightarrow \mathcal{D}^{\prime}(.) \hookrightarrow \mathcal{G}($.$) (where$ $\mathcal{D}^{\prime}$ denotes the space of distributions). $\hat{\iota}$ commutes with partial derivatives, and its restriction to $\mathcal{C}^{\infty}$ is a sheaf morphism of algebras. The map $\iota$ defined above also provides a linear embedding of $\mathcal{S}^{\prime}\left(\mathbb{R}^{n}\right)$ into $\mathcal{G}_{\tau}\left(\mathbb{R}^{n}\right)$ commuting with partial derivatives and making

$$
\mathcal{O}_{C}\left(\mathbb{R}^{n}\right)=\left\{f \in \mathcal{C}^{\infty}\left(\mathbb{R}^{n}\right): \exists p>0 \forall \alpha \in \mathbb{N}_{o}^{n} \sup _{x \in \mathbb{R}^{n}}(1+|x|)^{-p}\left|\partial^{\alpha} f(x)\right|<\infty\right\}
$$

a faithful subalgebra. So far, Colombeau algebras are the only known differential algebras enjoying these optimal embedding properties. Moreover, an intrinsic 
global formulation of Colombeau's construction on differentiable manifolds retaining all the characteristics of the local theory has recently been achieved $([12,13])$. Of the various variants of Colombeau algebras we shall also use the subalgebra $\mathcal{G}_{\infty}(\Omega)$ of $\mathcal{G}(\Omega)$ consisting of those elements of $\mathcal{G}(\Omega)$ possessing a representative $\left(u_{\varepsilon}\right)_{\varepsilon}$ such that $\sup _{\varepsilon \in I}\left\|u_{\varepsilon}\right\|_{L^{\infty}(\Omega)}<\infty$. Finally, we shall employ the "mixed type"-algebra $\tilde{\mathcal{G}}(\mathbb{R} \times \Omega)$ whose elements satisfy $\mathcal{G}$-bounds with respect to $t \in \mathbb{R}$ and $\mathcal{G}_{\tau}$-bounds with respect to $x \in \Omega$.

The ring of constants in $\mathcal{G}$ is denoted by $\overline{\mathbb{C}}$, its elements are called generalized numbers. Elements of $\overline{\mathbb{C}}$ may be used to model infinitesimal numbers (e.g., $(\varepsilon)_{\varepsilon \in I}$ is a representative of an infinitely small yet nonzero generalized number), which may be viewed as a "nonstandard" aspect of the theory. The support of a generalized function $U \in \mathcal{G}(\Omega)$, $\operatorname{supp}_{g} U$, is defined as the complement of the largest open subset $\Omega^{\prime}$ of $\Omega$ such that $U_{\mid \Omega^{\prime}}=0$. This notion is coherent with the embedding $\iota$, i.e. for any $T \in \mathcal{D}^{\prime}(\Omega)$ we have $\operatorname{supp} T=\operatorname{supp}_{g}(\iota(T))$. Finally, we mention the concept of association in the algebra $\mathcal{G}$ : Two elements $F, G$ are associated $F \approx G$ if there exist representatives $F_{\varepsilon}$ and $G_{\varepsilon}$ of $F$ and $G$, respectively, such that

$$
\lim _{\varepsilon \rightarrow 0} \int\left(G_{\varepsilon}(x)-F_{\varepsilon}(x)\right) \psi(x) d x=0, \text { for all } \psi \in \mathcal{D}(\Omega)
$$

Clearly, this definition does not depend on the choice of representatives. The concept of association (resp. strong association, cf. 3.2 below) plays a central role in the Colombeau framework as in many cases it allows for a distributional interpretation of results achieved in $\mathcal{G}$. Particularly in applications to physics and numerics it is of fundamental importance (cf. e.g. [7], [11], [36]).

With this terminology at hand we can now formulate the main goals of this article: Let $G$ be a transformation group acting on the space of independent and dependent variables of a system (11) of (linear or nonlinear) differential equations. We are looking for criteria for $G$ to transform weak solutions of (11) into weak solutions. More precisely, we shall develop conditions under which $G$ transforms $\mathcal{D}^{\prime}$-solutions to $\mathcal{D}^{\prime}$-solutions (in case (1) is linear), $\mathcal{G}$-solutions to $\mathcal{G}$-solutions, or solutions in the sense of association into solutions in the sense of association (in which case (11) is to be replaced by $\Delta\left(x, u^{(n)}\right) \approx 0$ ). We shall see that these questions are in fact closely linked and that criteria for one situation are often useful in other cases as well. As an application we study the symmetries of the quasilinear hyperbolic system $u_{t}+A(u) u_{x}=0$ where $A$ is an $s \times s$ matrix with $\mathcal{C}^{1}\left(\mathbb{R}^{s}\right)$ entries (cf. [30], [8]). For treating this system distribution type spaces are not convenient, while $\mathcal{G}$ provides a quite satisfactory solution concept (cf. [17, 26] and the literature cited therein). We consider strongly associated solutions and calculate symmetry transformations of such solutions. Also, we discuss infinitesimal criteria for symmetries of this system.

\section{Factorization properties, symmetries in $\mathcal{G}$}

Let $G$ be a projectable group action on some open set $\mathcal{M} \subset \mathcal{X} \times \mathcal{U}$. As was already pointed out in the introduction, composition in the framework of Colombeau generalized functions requires polynomial growth restrictions on the smooth functions. Thus we first single out those group actions which can be applied to elements of $\mathcal{G}$ (cf. [21]). An element $g \in G$ with $g \cdot(x, u)=$ 
$\left(\Xi_{g}(x), \Phi_{g}(x, u)\right)$, is called slowly increasing if $u \mapsto \Phi_{g}(x, u)$ is slowly increasing, uniformly for $x$ in compact sets; $g$ is called strictly slowly increasing if $\Phi_{g} \in \mathcal{O}_{M}(\mathcal{M})$.

If $\Omega \subset \mathcal{X}, U \in \mathcal{G}(\Omega)$ and $g$ is slowly increasing, the action of $g$ on $U$ is the element of $\mathcal{G}\left(\Xi_{g}(\Omega)\right)$ given by

$$
g U=\operatorname{cl}\left[\left(\Phi_{g} \circ\left(\mathrm{id} \times u_{\varepsilon}\right) \circ \Xi_{g}^{-1}\right)_{\varepsilon}\right]
$$

Also, in order to be able to insert elements of $\mathcal{G}(\Omega)$ into (1) we will from now on suppose that $u^{(n)} \mapsto \Delta\left(x, u^{(n)}\right)$ is slowly increasing, uniformly for $x$ in compact sets. A symmetry group of (1) in $\mathcal{G}$ is a local transformation group acting on $\mathcal{X} \times \mathcal{U}$ such that if $U$ is a solution of the system in $\mathcal{G}, g \in G$ and $g \cdot U$ is defined, then also $g \cdot U$ is a solution of $\Sigma$ in $\mathcal{G}$.

Let $G$ be a slowly increasing symmetry group of some differential equation

$$
\Delta\left(x, u^{(n)}\right)=0
$$

and let $U \in \mathcal{G}(\Omega)$ be a generalized solution to (3). Then for any representative $\left(u_{\varepsilon}\right)_{\varepsilon}$ of $U$ there exists some $\left(n_{\varepsilon}\right)_{\varepsilon} \in \mathcal{N}(\Omega)$ such that for all $x$ and all $\varepsilon$ we have

$$
\Delta\left(x, \operatorname{pr}^{(n)} u_{\varepsilon}(x)\right)=n_{\varepsilon}(x)
$$

Due to the nontrivial right hand side of (⿶) it is clear that a direct (componentwise) application of classical symmetry methods to Colombeau solutions is not feasible. A transfer of classical symmetry groups into the $\mathcal{G}$-setting therefore has to rely on properties of symmetry transformations that are better suited to the algebraic structure of $\mathcal{G}$. The key concept serving this purpose (and, as we shall see shortly, at the same time applicable to symmetries of $\mathcal{D}^{\prime}$ - and other weak solutions) is that of factorization:

Let $G=\left\{g_{\eta} \mid \eta \in \mathbb{R}\right\}$ be a (classical) one parameter symmetry group of (值)

$$
g_{\eta} \cdot(x, u)=\left(\Xi_{\eta}(x, u), \Phi_{\eta}(x, u)\right)
$$

Then by [28], Prop. 2.10 there exists a smooth map $Q: \mathcal{W} \rightarrow \mathbb{R}^{s^{2}}(\mathcal{W}$ open in $\left.\mathbb{R} \times \mathcal{M}^{(n)}, 0 \times \mathcal{M}^{(n)} \subseteq \mathcal{W}\right)$ such that

$$
\Delta\left(\operatorname{pr}^{(n)} g_{\eta}(z)\right)=Q(\eta, z) \Delta(z)
$$

Throughout this paper, $\mathcal{W}$ will denote an open set as specified above. By [21], Th. 3.4 for any smooth $u: \Omega \subset \mathbb{R}^{p} \rightarrow \mathbb{R}^{q}$ such that $g_{\eta} u$ exists we have

$$
\begin{aligned}
& \Delta\left(\Xi_{\eta}(x, u(x)), \mathrm{pr}^{(n)}\left(g_{\eta} u\right)\left(\Xi_{\eta}(x, u(x))\right)\right) \\
& =Q\left(\eta, x, \operatorname{pr}^{(n)} u(x)\right) \cdot \Delta\left(x, \operatorname{pr}^{(n)} u(x)\right) .
\end{aligned}
$$

In particular, $\eta \rightarrow g_{\eta}$ is a symmetry group of (传) in $\mathcal{G}$ if $\left(x, u^{(n)}\right) \rightarrow Q_{\mu \nu}(\eta, x$, $\left.\operatorname{pr}^{(n)} u(x)\right)$ is slowly increasing with respect to $u^{(n)}$, uniformly for $x$ varying in compact sets (21], Proposition 3.5). Thus the need for conditions ensuring that the $Q_{\mu \nu}$ remain well behaved (in the above sense) arises. Theorem 3.8 of [21] shows that for scalar differential equations possessing a stand alone term (i.e. $\frac{\partial \Delta}{\partial z_{k}}=c$ for some $k>p$ ) this is indeed always the case. Our first aim is to generalize this result.

Since (11) is nondegenerate it follows that the Jacobian $J(\Delta)$ of $\Delta$ has rank $s$ on the zero-set of $\Delta$. The following result uses a mild strengthening of this assumption to derive a factorization property adapted to the polynomial growth restrictions necessary for applying nonlinearities to elements of $\mathcal{G}$. 
2.1 Theorem Let $G=\left\{g_{\eta} \mid \eta \in\left(-\eta_{0}, \eta_{0}\right)\right\}$ be a slowly increasing symmetry group of system (1) and suppose that there exist $p<k_{1}<\cdots<k_{s} \leq N$ such that, setting $J_{k_{1}, \ldots, k_{s}}(\Delta):=\left(\frac{\partial \Delta_{i}}{\partial z_{k_{j}}}\right)_{i, j=1 \ldots s}$, the following conditions are satisfied:

(i) $z \rightarrow\left(z^{\prime}, \Delta(z)\right)$ is injective, where $z^{\prime}=\left(z_{1}, \ldots, \widehat{z_{k_{1}}}, \ldots, \widehat{z_{k_{s}}}, \ldots, z_{N}\right)$.

(ii) $z \rightarrow\left(\operatorname{det}\left(J_{k_{1}, \ldots, k_{s}}(\Delta)\right)\right)^{-1}(z)$ is defined globally and is slowly increasing, uniformly for $\left(z_{1}, \ldots, z_{p}\right)$ varying in compact sets.

Then there exists a smooth mapping $Q: \mathcal{W} \rightarrow \mathbb{R}^{s^{2}}$ which is slowly increasing in $z \in \mathcal{M}^{(n)}$, uniformly for $z_{1}, \ldots, z_{p}$ varying in compact sets such that (5) holds. In particular, (6) holds for any smooth $u: \Omega \subseteq \mathbb{R}^{p} \rightarrow \mathbb{R}^{q}$ such that $g_{\eta} u$ exists.

Proof. Without loss of generality we may suppose $\left\{k_{1}, \ldots, k_{s}\right\}=\{N-s+$ $1, \ldots, N\}$. We set $z^{\prime}=\left(z_{1}, \ldots, z_{N-s}\right), z^{\prime \prime}=\left(z_{N-s+1}, \ldots, z_{N}\right)$ and define $\tilde{\Delta}: \mathbb{R}^{N} \rightarrow \mathbb{R}^{N}$ by

$$
\tilde{\Delta}(z)=\left(z^{\prime}, \Delta(z)\right)
$$

Since $\operatorname{det}(J(\tilde{\Delta}))=\operatorname{det}\left(J_{k_{1}, \ldots, k_{s}}(\Delta)\right) \neq 0, \tilde{\Delta}$ is a diffeomorphism by (i). Moreover,

$$
\Delta \circ \tilde{\Delta}^{-1}=\left(y_{1}, \ldots, y_{N}\right) \rightarrow\left(y_{N-s+1}, \ldots, y_{N}\right)=y \rightarrow y^{\prime \prime}
$$

Now set $f_{\eta}(z)=\Delta\left(\operatorname{pr}^{(n)} g_{\eta}(z)\right)$. By [21], Proposition 3.7 and our general assumption on $\Delta, f_{\eta}$ is slowly increasing in $z$, uniformly for $\left(z_{1}, \ldots, z_{p}\right)$ varying in compact sets. Since $\eta \rightarrow g_{\eta}$ is a symmetry group of (1) we have $f_{\eta} \circ \tilde{\Delta}^{-1}(y) \equiv 0$ if $y^{\prime \prime} \equiv 0$. Thus

$$
\begin{aligned}
f_{\eta} \circ \tilde{\Delta}^{-1}(y) & =\left.\left(f_{\eta} \circ \tilde{\Delta}^{-1}\right)\left(y^{\prime}, \tau y^{\prime \prime}\right)\right|_{\tau=0} ^{1} \\
& =\int_{0}^{1} \frac{d}{d \tau}\left(f_{\eta} \circ \tilde{\Delta}^{-1}\right)\left(y^{\prime}, \tau y^{\prime \prime}\right) d \tau \\
& =\left(\int_{0}^{1} J_{k_{1} \ldots k_{s}}\left(f_{\eta} \circ \tilde{\Delta}^{-1}\right)\left(y^{\prime}, \tau y^{\prime \prime}\right) d \tau\right) \cdot y^{\prime \prime}
\end{aligned}
$$

Inserting $z=\tilde{\Delta}^{-1}(y)$ into (7) we arrive at

$$
f_{\eta}(z)=\left(\int_{0}^{1} J_{k_{1}, \ldots, k_{s}}\left(f_{\eta} \circ \tilde{\Delta}^{-1}\right)\left(z^{\prime}, \tau \Delta(z)\right) d \tau\right) \cdot \Delta(z)=: Q(\eta, z) \cdot \Delta(z)
$$

In order to establish the claimed growth properties of $Q$, since $f_{\eta}$ and $\Delta$ are slowly increasing, by the chain rule it suffices to estimate $J_{k_{1} \ldots k_{s}}\left(\tilde{\Delta}^{-1}\right)$. The determinant of this Jacobian is precisely $\left(\operatorname{det}\left(J_{k_{1}, \ldots, k_{s}}(\Delta)\right)\right)^{-1}$, so the claim follows from (ii).

\subsection{Remarks}

(i) By dropping the growth restrictions on $\Delta, G$ (in particular, allowing for $G$ to be nonprojectable and merely supposing that $\left(\operatorname{det}\left(J_{k_{1}, \ldots, k_{s}}(\Delta)\right)\right)^{-1}$ exists globally), the same proof as above still provides the explicit form (8) of the factorization property of general symmetry groups of (11), which reads

$$
\begin{aligned}
\Delta\left(\operatorname{pr}^{(n)} g_{\eta}(z)\right)=( & \left(\int _ { 0 } ^ { 1 } J _ { k _ { 1 } , \ldots , k _ { s } } ( f _ { \eta } \circ \tilde { \Delta } ^ { - 1 } ) \left(z_{1}, \ldots, z_{k_{1}-1}, \tau \Delta_{1}(z), z_{k_{1}+1},\right.\right. \\
& \left.\left.\ldots, z_{k_{s}-1}, \tau \Delta_{s}(z), z_{k_{s}+1}, \ldots, z_{N}\right) d \tau\right) \cdot \Delta(z)
\end{aligned}
$$


(ii) A necessary and sufficient condition for (ii) in 2.1 is given by

$$
\begin{aligned}
& \forall K \subset \subset \mathbb{R}^{p} \exists C>0 \exists r>0 \forall z \in \mathcal{M}^{(n)}: \\
& \inf _{\left(z_{1}, \ldots, z_{p}\right) \in K}\left|\frac{\partial\left(\Delta_{1}, \ldots, \Delta_{s}\right)}{\partial\left(z_{k_{1}}, \ldots, z_{k_{s}}\right)}(z)\right| \geq C\left(\left(1+\left|z_{p+1}\right|\right) \ldots\left(1+\left|z_{N}\right|\right)\right)^{-r}
\end{aligned}
$$

(iii) An extensive compilation of sufficient conditions for global injectivity of smooth maps ( $\tilde{\Delta}$ in our case) can be found in [29]. As an example we mention a result of Gale and Nikaido ([29], ch. 3) stating that any $F$ : $\Omega \rightarrow \mathbb{R}^{n}$ (with $\Omega$ a rectangular region in $\mathbb{R}^{n}$ ) is injective if its Jacobian is a $P$-matrix on all of $\Omega$ (i.e. all principal minors are positive). Moreover, global invertibility results for Sobolev functions are given in [2].

2.3 Corollary Under the assumptions of Theorem 2.1, $G$ is a symmetry group of (因) in $\mathcal{G}$.

Proof. Let $u \in \mathcal{G}(\Omega)^{q}$ be a solution of (11). Then by (6), for any representative $\left(u_{\varepsilon}\right)_{\varepsilon \in I}$ of $u$ we have, taking into account the projectability of $g_{\eta}$,

$$
\begin{aligned}
& \Delta\left(x, \operatorname{pr}^{(n)}\left(g_{\eta} u_{\varepsilon}\right)(x)\right) \\
& =Q\left(\eta, \Xi_{-\eta}(x), \operatorname{pr}^{(n)} u_{\varepsilon}\left(\Xi_{-\eta}(x)\right)\right) \cdot \Delta\left(\Xi_{-\eta}(x), \operatorname{pr}^{(n)} u_{\varepsilon}\left(\Xi_{-\eta}(x)\right)\right)
\end{aligned}
$$

Here, $\Delta\left(\Xi_{-\eta}(x), \operatorname{pr}^{(n)} u_{\varepsilon}\left(\Xi_{-\eta}(x)\right)\right)$ is negligible by definition and Theorem 2.1 shows $\operatorname{cl}\left[x \rightarrow Q\left(\eta, \Xi_{-\eta}(x), \operatorname{pr}^{(n)} u_{\varepsilon}\left(\Xi_{-\eta}(x)\right)\right)\right]$ to be moderate. It follows that $\left(\Delta\left(x, \operatorname{pr}^{(n)}\left(g_{\eta} u_{\varepsilon}\right)(x)\right)\right)_{\varepsilon \in I}$ is negligible, which precisely means that $g_{\eta} u$ is a solution in $\mathcal{G}$.

2.4 Example Suppose that system (11) satisfies $\frac{\partial \Delta_{i}}{\partial z_{j}}=c_{i} \delta_{i k_{j}}$ for some $p<$ $k_{1}<\cdots<k_{s} \leq N$ and nonzero constants $c_{i}$. Then (1) satisfies conditions (i) and (ii) of Theorem 2.1. In fact, $\operatorname{det}\left(J_{k_{1}, \ldots, k_{s}}(\Delta)\right)$ is constant and since the underlying domain is convex the above assumption on (11) is equivalent with

$$
\Delta_{i}(z)=c_{i} z_{k_{i}}+F_{i}\left(z^{\prime}\right) \quad(1 \leq i \leq s)
$$

with $F_{i}$ smooth and $z^{\prime}$ as in 2.1 (i). From this, injectivity of $z \rightarrow\left(z^{\prime}, \Delta(z)\right)$ is immediate. Thus by 2.1 any slowly increasing classical symmetry group of (1) is a $\mathcal{G}$-symmetry group as well. This observation applies e.g. to the system

$$
\begin{aligned}
U_{t}+U U_{x} & =0 \\
V_{t}+U V_{x} & =0
\end{aligned}
$$

considered in [21], Example 3.6. Also, Theorem 3.8 of 21] is a special case of this setup (for $s=1$ ).

\section{Associated and distributional symmetry groups}

In this section we are going to investigate symmetries of system (11) in the sense of association, i.e. we shall be concerned with group actions that transform solutions of

$$
\Delta_{i}\left(x, u^{(n)}\right) \approx 0 \quad(i=1, \ldots, s)
$$


into solutions in the sense of association. Such group actions will be called symmetries in the sense of association or, by slight abuse of terminology, associated symmetries. Also, we will give a rather general criterion for transferring classical symmetry groups of linear systems to the distributional setting. In what follows we will only consider projectable symmetry groups.

3.1 Definition $u=\left(u_{1}, \ldots, u_{q}\right) \in \mathcal{G}(\Omega)^{q}$ is a solution to (12) (also called an associated solution to (1)) if $u$ has a representative $\left(u_{1 \varepsilon}, \ldots, u_{q \varepsilon}\right) \in \mathcal{E}_{M}(\Omega)^{q}$ such that for every $\varphi \in \mathcal{D}(\Omega)$

$$
\int \Delta_{i}\left(x, \operatorname{pr}^{(n)} u_{\varepsilon}(x)\right) \varphi(x) d x \rightarrow 0 \text { as } \varepsilon \rightarrow 0,1 \leq i \leq s
$$

The set of all associated solutions to (1) will be denoted by $\mathcal{A}_{\Delta}$. The set of all $u \in\left(\mathcal{G}_{\infty}\right)^{q}$ satisfying (13) will be termed $\mathcal{A B}_{\Delta}$. Let $\mathcal{A} \subseteq \mathcal{A}_{\Delta}$. A symmetry group $\eta \rightarrow g_{\eta}$ of (1) will be called an $\mathcal{A}$-symmetry group if $g_{\eta} U \in \mathcal{A}$ whenever $U \in \mathcal{A}$ and $g_{\eta} U$ is defined.

In what follows, $\mathcal{C}_{c}^{k}(\Omega)\left(k \in \mathbb{N}_{0}\right)$, the space of compactly supported $\mathcal{C}^{k}$-functions on $\Omega$ is equipped with the inductive limit topology of its subspaces $\mathcal{C}_{K}^{k}(\Omega)=$ $\left\{f \in \mathcal{C}^{k}(\Omega) \mid \operatorname{supp} f \subseteq K\right\}(K$ compact in $\Omega)$.

3.2 Definition Let $u=\left(u_{1}, \ldots, u_{q}\right) \in \mathcal{A}_{\Delta}(\Omega)$ (resp. $u \in \mathcal{A B}_{\Delta}(\Omega)$ ) and let $k \in \mathbb{N}_{0} . u$ is called a $k$-strongly associated $(\stackrel{k}{\approx}$-associated) solution to (耳) if it has a representative $\left(u_{1 \varepsilon}, \ldots, u_{q \varepsilon}\right) \in \mathcal{E}_{M}(\Omega)^{q}$ such that for every set $B \subseteq \mathcal{C}_{c}^{\infty}(\Omega)$ which is bounded in $\mathcal{C}_{c}^{k}(\Omega)$ we have

$$
\lim _{\varepsilon \rightarrow 0} \sup _{\varphi \in B} \int \Delta_{i}\left(x, \operatorname{pr}^{(n)} u_{\varepsilon}(x)\right) \varphi(x) d x=0 \quad(1 \leq i \leq s)
$$

The space of $k$-strongly associated solutions to (11) is denoted by $\mathcal{A S}_{\Delta}^{k}$ and we set $\mathcal{A B S}_{\Delta}^{k}:=\mathcal{A S}_{\Delta}^{k} \cap \mathcal{G}_{\infty}$. The corresponding symmetry groups are called $\mathcal{A S}_{\Delta}^{k}$ and $\mathcal{A B S}_{\Delta}^{k}$-symmetry groups, respectively.

3.3 Remark Associated solutions (i.e., solutions of type $\mathcal{A}_{\Delta}$ ) play a central role in applications to numerics (cf. the remarks in Section 1). Moreover, by imposing slight changes in their definition, Colombeau type algebras can be adapted to a wide variety of solution types. Thus $\mathcal{G}_{\infty}$ is particularly useful for the investigation of shock wave solutions of conservation laws (cf. 3.4, 3.6, 3.7 below), which fall into this class of generalized functions. It will turn out in Theorem 3.5 (i) that the uniform bounddedness of solutions is essential for the characterization of $\mathcal{A B S}_{\Delta}$-symmetry groups.

3.4 Example Consider the Riemann problem

$$
\begin{aligned}
& u_{t}+u u_{x}=0 \\
& u(x, 0)=u_{l}+\left(u_{r}-u_{l}\right) H(x)
\end{aligned}
$$

where $H$ denotes the Heaviside function. For $u_{l}>u_{r}$ the unique weak solution to this problem is $u(x, t)=u_{l}+\left(u_{r}-u_{l}\right) H(x-c t),(x, t) \in \mathbb{R} \times[0, \infty)$, where 
$c=\left(u_{l}+u_{r}\right) / 2$. Let $\theta \in \mathcal{D}(\mathbb{R}), \theta \geq 0, \int \theta=1$ and set $H_{\varepsilon}(y)=\int_{-\infty}^{y / \varepsilon} \theta(x) d x$, $\delta_{\varepsilon}(y)=\frac{1}{\varepsilon} \theta\left(\frac{y}{\varepsilon}\right)$. We are looking for 1-strongly associated solutions to (14) of the form $u_{\varepsilon}(x, t)=u_{l}+\left(u_{r}-u_{l}\right) H_{\varepsilon}(x-c t)$, with $c$ to be determined. Thus let $B$ be a bounded subset of $\mathcal{C}_{c}^{1}(\mathbb{R} \times(0, \infty))$. This means that there exists $K \subset \subset \mathbb{R} \times(0, \infty)$ such that $\operatorname{supp} \varphi \subseteq K$ for all $\varphi \in B$ and

$$
\sup \left\{\left|\partial^{\alpha} \varphi(x, t)\right||\varphi \in B,(x, t) \in K,| \alpha \mid \leq 1\right\}<\infty
$$

Let $\psi \in B$. Then

$$
\begin{gathered}
\int_{\mathbb{R} \times(0, \infty)}\left[u_{\varepsilon t}(x, t)+u_{\varepsilon}(x, t) u_{\varepsilon x}(x, t)\right] \psi(x, t) d x d t= \\
\int_{\mathbb{R} \times(0, \infty)}\left[-c\left(u_{r}-u_{l}\right) \partial_{x} H_{\varepsilon}(x-c t)+\right. \\
\left.\frac{1}{2} \partial_{x}\left(\left(u_{l}+\left(u_{r}-u_{l}\right) H_{\varepsilon}(x-c t)\right)^{2}\right)\right] \psi(x, t) d x d t \\
=-\int_{\mathbb{R} \times(0, \infty)}\left[-c\left(u_{r}-u_{l}\right) H_{\varepsilon}(x-c t)+\frac{1}{2}\left(\left(2 u_{l}\left(u_{r}-u_{l}\right)\right.\right.\right. \\
\left.\left.\left.\cdot H_{\varepsilon}(x-c t)+\left(u_{r}-u_{l}\right)^{2} H_{\varepsilon}(x-c t)\right)^{2}\right)\right] \psi_{x}(x, t) d x d t \\
\rightarrow \int_{0}^{\infty}\left[c\left(u_{r}-u_{l}\right)-\frac{1}{2}\left(u_{r}^{2}-u_{l}^{2}\right)\right] \psi(c t, t) d t
\end{gathered}
$$

by dominated convergence, uniformly for $\psi \in B$. Thus $u_{\varepsilon}$ as above is a 1strongly associated solution if and only if $c=\left(u_{l}+u_{r}\right) / 2$, i.e. if and only if the Rankine-Hugoniot jump condition is satisfied.

The transfer of classical symmetry groups into symmetry groups in the sense of association is again governed by factorization properties. Sufficient conditions for this transfer are provided by the following result:

3.5 Theorem Let $G=\left\{g_{\eta} \mid \eta \in\left(-\eta_{0}, \eta_{0}\right)\right\}$ be a slowly increasing symmetry group of (11) admitting a global factorization of the form (5). Then

(i) If $Q$ depends exclusively on $\eta, x$ and $u$ then $g_{\eta}$ is an $\mathcal{A B S}_{\Delta}$-symmetry group of (17).

(ii) If $Q$ depends exclusively on $x$ and $\eta$ then for any $k>0 g_{\eta}$ is an $\mathcal{A S}_{\Delta}^{k}$ symmetry group of (1).

Proof. (i) Let $\phi \in \mathcal{D}\left(\Xi_{\eta}(\Omega)\right)$ and $u \in \mathcal{A B S}_{\Delta}(\Omega)$. It follows from (2) that $u \in \mathcal{G}_{\infty}\left(\Xi_{\eta}(\Omega)\right)^{q}$. Moreover, by (6), for any $1 \leq i \leq s$ we have

$$
\begin{aligned}
& \int \Delta_{i}\left(x, \operatorname{pr}^{(n)}\left(g_{\eta} u_{\varepsilon}\right)(x)\right) \phi(x) d x \\
& =\sum_{j} \int Q_{i j}\left(\eta, \Xi_{-\eta}(x), u_{\varepsilon}\left(\Xi_{-\eta}(x)\right)\right) \Delta_{j}\left(\Xi_{-\eta}(x), \operatorname{pr}^{(n)} u_{\varepsilon}\left(\Xi_{-\eta}(x)\right)\right) \phi(x) d x \\
& =\sum_{j} \int \Delta_{j}\left(x^{\prime}, \operatorname{pr}^{(n)} u_{\varepsilon}\left(x^{\prime}\right)\right) \underbrace{Q_{i j}\left(\eta, x^{\prime}, u_{\varepsilon}\left(x^{\prime}\right)\right) \phi\left(\Xi_{\eta}\left(x^{\prime}\right)\right)\left|\operatorname{det} D \Xi_{\eta}\left(x^{\prime}\right)\right|}_{(*)} d x^{\prime}
\end{aligned}
$$


If $\phi$ varies in a subset of $\mathcal{D}\left(\Xi_{\eta}(\Omega)\right)$ which is bounded in $\mathcal{C}_{c}$ then due to the fact that the $u_{\varepsilon}$ are globally bounded, $(*)$ varies in a $\mathcal{C}_{c}$-bounded subset of $\mathcal{D}(\Omega)$. Hence the above expression tends to zero, uniformly in $\phi$ and $\varepsilon$.

(ii) With $\phi$ as in (i),

$$
\begin{aligned}
& \int \Delta_{i}\left(x, \operatorname{pr}^{(n)}\left(g_{\eta} u_{\varepsilon}\right)(x)\right) \phi(x) d x \\
& =\sum_{j} \int \Delta_{j}\left(x^{\prime}, \operatorname{pr}^{(n)} u_{\varepsilon}\left(x^{\prime}\right)\right) \underbrace{Q_{i j}\left(\eta, x^{\prime}\right) \phi\left(\Xi_{\eta}\left(x^{\prime}\right)\right)\left|\operatorname{det} D \Xi_{\eta}\left(x^{\prime}\right)\right|}_{(*)} d x^{\prime}
\end{aligned}
$$

which tends to zero since $(*)$ is $\mathcal{C}_{c}^{k}$-bounded if $\phi$ varies in a $\mathcal{C}_{c}^{k}$-bounded subset of $\mathcal{D}\left(\Xi_{\eta}(\Omega)\right)$.

3.6 Example Let $A$ be an $s \times s$ matrix with $\mathcal{C}^{1}$-entries $a^{i j}=a^{i j}\left(u^{1}, \ldots, u^{s}\right), i, j$ $=1, \ldots, s$ on $\mathbb{R}^{s}$. We consider the quasilinear system

$$
\Delta\left(u, u_{x}, u_{t}\right) \equiv u_{t}+A(u) u_{x}
$$

(for solvability resp. unique solvability of (15) we refer to [30] and [8]) and the action of a classical symmetry group $G=\left\{g_{\eta} \mid \eta \in\left(-\eta_{0}, \eta_{0}\right)\right\}$

$$
\left.\begin{array}{rl}
\tilde{x} & =\Xi_{\eta}^{1}(x, t) \\
\tilde{t} & =\Xi_{\eta}^{2}(x, t) \\
\tilde{u} & =\Phi_{\eta}(x, t, u)
\end{array}\right\}, \quad \eta \in\left(-\eta_{0}, \eta_{0}\right)
$$

where $\Xi_{\eta}^{1}, \Xi_{\eta}^{2} \in \mathcal{C}^{\infty}(\Omega), \Phi_{\eta} \in\left(\mathcal{C}^{\infty}\left(\Omega \times \mathbb{R}^{s}\right)\right)^{s}, \eta \in\left(-\eta_{0}, \eta_{0}\right)$. We are going to show that $G$ is an $\mathcal{A S}_{\Delta}^{k}$-symmetry group of (15) for each $k$ provided that

(i) $\partial_{u} \Phi_{\eta}$ does not depend on $u$, i.e. $G$ acts linearly on the dependent variables.

(ii) $\Xi_{\eta}^{2}$ does not depend on $x$.

Denoting as above the Jacobian of $\Xi_{\eta}$ by $J \Xi_{\eta}, \tilde{u}_{\tilde{x}}$ and $\tilde{u}_{\tilde{t}}$ are given by the first and second column of the matrix valued function $\Phi_{\eta}^{1} \in\left(\mathcal{C}^{\infty}\left(\Omega \times \mathbb{R}^{3 s}\right)\right)^{s \times 2}$, where $\Phi_{\eta}^{1}\left(x, t, u, u_{x}, u_{t}\right)$ is defined by

$$
\left(\left[\Phi_{\eta x}(x, t, u) \quad \Phi_{\eta t}(x, t, u)\right]+\frac{\partial \Phi_{\eta}}{\partial u}(x, t)\left[\begin{array}{ll}
u_{x} & u_{t}
\end{array}\right]\right)\left(J \Xi_{\eta}(x, t)\right)^{-1}
$$

Moreover,

$$
\begin{gathered}
\Delta\left(\tilde{u}, \tilde{u}_{\tilde{x}}, \tilde{u}_{\tilde{t}}\right)=: \tilde{\Delta}_{\eta}\left(x, t, u, u_{x}, u_{t}\right) \\
=\int_{0}^{1}\left(\frac{\partial \tilde{\Delta}_{\eta}}{\partial u_{t}}\right)\left(x, t, u, u_{x}, \theta u_{t}+(1-\theta)\left(-A(u) u_{x}\right)\right) d \theta \times\left(u_{t}-\left(-A(u) u_{x}\right)\right) \\
=Q_{\eta}(x, t, u) \Delta\left(u, u_{x}, u_{t}\right),
\end{gathered}
$$

Note that $Q_{\eta}$ does not depend on $u_{x}, u_{t}$ by our assumption on $G$ and the explicit form of $\Delta$. Now

$$
\tilde{\Delta}_{\eta}=\Phi_{\eta}^{1}\left(x, t, u, u_{x}, u_{t}\right)\left[\begin{array}{l}
0 \\
1
\end{array}\right]+A\left(\Phi_{\eta}(x, t, u)\right) \Phi_{\eta}^{1}\left(x, t, u, u_{x}, u_{t}\right)\left[\begin{array}{l}
1 \\
0
\end{array}\right]
$$




$$
\begin{aligned}
\Phi_{\eta}^{1}\left[\begin{array}{l}
0 \\
1
\end{array}\right] & =\left(\left[\begin{array}{ll}
\Phi_{\eta x} & \Phi_{\eta t}
\end{array}\right]+\Phi_{\eta u}\left[\begin{array}{ll}
u_{x} & u_{t}
\end{array}\right]\right) \frac{1}{\operatorname{det} J \Xi_{\eta}}\left[\begin{array}{c}
-\Xi_{\eta t}^{1} \\
\Xi_{\eta x}^{1}
\end{array}\right] \\
& =\frac{1}{\operatorname{det} J \Xi_{\eta}}\left(\Xi_{\eta x}^{1} \Phi_{\eta t}-\Xi_{\eta t}^{1} \Phi_{\eta x}+\Xi_{\eta x}^{1} \Phi_{\eta u} u_{t}-\Xi_{\eta t}^{1} \Phi_{\eta u} u_{x}\right) \\
\Phi_{\eta}^{1}\left[\begin{array}{l}
1 \\
0
\end{array}\right] & \left.=\left(\begin{array}{ll}
{\left[\Phi_{\eta x}\right.} & \Phi_{\eta t}
\end{array}\right]+\Phi_{\eta u}\left[\begin{array}{ll}
u_{x} & u_{t}
\end{array}\right]\right) \frac{1}{\operatorname{det} J \Xi_{\eta}}\left[\begin{array}{c}
\Xi_{\eta t}^{2} \\
-\Xi_{\eta x}^{2}
\end{array}\right] \\
& =\frac{1}{\operatorname{det} J \Xi_{\eta}}\left(\Xi_{\eta t}^{2} \Phi_{\eta x}-\Xi_{\eta x}^{2} \Phi_{\eta t}+\Xi_{\eta t}^{2} \Phi_{\eta u} u_{x}-\Xi_{\eta x}^{2} \Phi_{\eta u} u_{t}\right)
\end{aligned}
$$

Thus

$$
Q_{\eta}=\frac{\partial \tilde{\Delta}_{\eta}}{\partial u_{t}}=\frac{1}{\operatorname{det} J \Xi_{\eta}}\left(\Xi_{\eta x}^{1} \Phi_{\eta u}-\Xi_{\eta x}^{2} A\left(\Phi_{\eta}\right) \Phi_{\eta u}\right)=\frac{\Xi_{\eta x}^{1} I_{s}-\Xi_{\eta x}^{2} A\left(\Phi_{\eta}\right)}{\Xi_{\eta x}^{1} \Xi_{\eta t}^{2}-\Xi_{\eta x}^{2} \Xi_{\eta t}^{1}} \Phi_{\eta u}
$$

where $\mathrm{I}_{s}$ is the $s \times s$ identity matrix. This implies

$$
\tilde{\Delta}_{\eta}\left(x, t, u, u_{x}, u_{t}\right)=\frac{\Xi_{\eta x}^{1} \mathrm{I}_{s}-\Xi_{\eta x}^{2} A\left(\Phi_{\eta}\right)}{\Xi_{\eta x}^{1} \Xi_{\eta t}^{2}-\Xi_{\eta t}^{1} \Xi_{\eta x}^{2}} \Phi_{\eta u} \Delta\left(x, t, u, u_{x}, u_{t}\right)=Q_{\eta} \Delta .
$$

By assumptions (i) and (ii) it follows that $Q_{\eta}$ does not depend on $u$. Thus our claim follows from 3.5 (ii).

3.7 Example Let $f \in \mathcal{C}^{1}(\mathbb{R})$ and let $F$ be a primitive of $f$. The generalized Burgers equation

$$
\begin{aligned}
& u_{t}+f(u) u_{x}=0 \\
& u(x, 0)=u_{l}+\left(u_{r}-u_{l}\right) H(x)
\end{aligned}
$$

has a weak solution of the form

$$
u(x, t)=u_{l}+\left(u_{r}-u_{l}\right) H(x-c t), \quad(x, t) \in \mathbb{R} \times(0, T)
$$

where $c=\left(F\left(u_{r}\right)-F\left(u_{l}\right)\right) /\left(u_{r}-u_{l}\right)$. (If $f^{\prime}>0$ and $u_{l}>u_{r}$, then this solution is unique.) The same arguments as in the case of Burgers' equation in 3.4 imply that (21) is the 1-strongly associated solution to (20) if and only if $c$ is of the given form. In this special case of (15), conditions (i) and (ii) of 3.6 are in fact necessary for shock wave solutions to be transformed into 1-strongly associated solutions. In fact, if (i) or (ii) is violated then from the explicit calculations in 3.6 it follows that the integrand in the proof of 3.5 (ii) will contain unbounded terms.

More complex factorizations arise in case $f$ is invertible. Indeed, by a straightforward explicit calculation, the infinitesimal generators

$$
\begin{aligned}
& \mathbf{w}_{1}=x t \partial_{x}+t^{2} \partial_{t}+\frac{1}{f^{\prime}(u)}(x-f(u) t) \partial_{u} \\
& \mathbf{w}_{2}=x^{2} \partial_{x}+x t \partial_{t}+\frac{f(u)}{f^{\prime}(u)}(x-f(u) t) \partial_{u}
\end{aligned}
$$

give rise to the group actions

$$
G_{1}: \tilde{x}=x(\eta)=\frac{x}{1-\eta t}, \tilde{t}=t(\eta)=\frac{t}{1-\eta t}, \tilde{u}=f^{-1}(\eta x+f(u)-\eta f(u) t)
$$




$$
G_{2}: \tilde{x}=x(\eta)=\frac{x}{1-\eta x}, \tilde{t}=t(\eta)=\frac{t}{1-\eta x}, \tilde{u}=f^{-1}\left(\frac{f(u)}{1-\eta(x-f(u) t)}\right)
$$

with factors

$$
\begin{aligned}
Q_{1} & =\frac{(1-\eta t)^{3} f^{\prime}}{f^{\prime}\left(f^{-1}(\eta x+f(u)-\eta f(u) t)\right)} \\
Q_{2} & =\frac{(1-\eta x)^{3} f^{\prime}(u)}{(1-\eta(x-t f))^{3} f^{\prime}\left(f^{-1}\left(\frac{f(u)}{1-\eta(x-t f(u))}\right)\right)}
\end{aligned}
$$

Theorem 3.5 raises the question of finding criteria for classical symmetry groups to display the favorable factorization properties used above. As an important case where a general result is available we now turn to systems of linear PDEs. With a view to applications in distribution theory (cf. section 4) we also restrict our attention to group actions which act linearly on the dependent variables.

3.8 Theorem Suppose that in (1), $\Delta$ is a linear differential operator:

$$
\Delta_{i}(z)=\sum_{k=p+1}^{N} a_{k}^{i}\left(z_{1}, \ldots, z_{p}\right) z_{k}+a_{0}^{i}\left(z_{1}, \ldots, z_{p}\right) \quad(1 \leq i \leq s)
$$

Furthermore, let $g_{\eta}$ be a one parameter symmetry group of (29) which acts linearly in the dependent variables:

$$
g_{\eta}(x, u)=\left(\Xi_{\eta}(x), \Phi_{\eta}(x) \cdot u+\Psi_{\eta}(x)\right)
$$

If there exist $p<k_{1}<\cdots<k_{s} \leq N$ such that $J_{k_{1}, \ldots, k_{s}}(\Delta)$ is globally nonsingular then conditions (i) and (ii) of 2.1 are satisfied and $Q$ in (5) depends exclusively on $\eta$ and $x=\left(z_{1}, \ldots, z_{p}\right)$.

Proof. Using the same notations and conventions as in the proof of Theorem 2.1 we have $\tilde{\Delta}(z)=\left(z_{1}, \ldots, z_{N-s}, A(x) \cdot z+a_{0}(z)\right)$ where the $s \times N$ matrix $A(x)$ is of the form $\left(A^{\prime}(x), A^{\prime \prime}(x)\right)$ with $A^{\prime}(x)$ an $s \times(N-s)$ matrix and $A^{\prime \prime}(x)=$ $J_{k_{1}, \ldots, k_{s}}(\Delta)$ invertible.

Thus

$$
\tilde{\Delta}^{-1}(y)=\left(y^{\prime}, A^{\prime \prime}\left(y_{1}, \ldots, y_{p}\right)^{-1} \cdot\left[y^{\prime \prime}-A^{\prime}\left(y_{1}, \ldots, y_{p}\right) \cdot y^{\prime}-a_{0}\left(y_{1}, \ldots, y_{p}\right)\right]\right)
$$

is affine linear in $y_{k}$ for $k>p$. By 23] and [28], (2.18) we have

$$
\operatorname{pr}^{(n)} g_{\eta}(z)=\left(\Xi_{\eta}(x), \Phi_{\eta}(x) z_{p+1}+\Psi_{\eta}(x), \ldots, \bar{z}_{k}, \ldots, \bar{z}_{N}\right)
$$

where

$$
\bar{z}_{k}=\sum_{l=p+1}^{N} b_{l}^{k}(\eta, x) z_{l}+b_{0}^{k}(\eta, x)
$$

with certain smooth functions $b_{l}^{k}$ (Actually, the upper limit $N$ in these sums is only attained for terms corresponding to highest order derivatives but for the following argument only the general form of (26) is of interest). Hence both $f_{\eta}$ and $\tilde{\Delta}^{-1}$ are (affine) linear in $z_{k}$ for $k>p$. It follows that the matrix $J_{k_{1}, \ldots, k_{s}}\left(f_{\eta} \circ \tilde{\Delta}^{-1}\right)$ is independent of $z_{k}$ for $k>p$. This observation, together with (8), finishes the proof. 
3.9 Remark The conclusion of Theorem 3.8 remains valid for a semilinear system

$$
\Delta_{i}(z)=\sum_{k=p+1}^{N} a_{k}^{i}\left(z_{1}, \ldots, z_{p}\right) z_{k}+a_{0}^{i}\left(z_{1}, \ldots, z_{p}, z_{p+1}, \ldots, z_{p+q}\right) \quad(1 \leq i \leq s)
$$

provided that $k_{1}, \ldots, k_{s}$ correspond to indices of highest order derivatives of the dependent variables. This follows immediately from an inspection of the above proof (only the form of $a_{0}$ in (24) changes).

3.10 Corollary Let $G$ be a symmetry group of the linear (resp. semilinear) system (22) (resp. (27)) such that the assumptions of Theorem 3.8 (resp. of Remark 3.9) are satisfied. Then $G$ is an $\mathcal{A S}_{\Delta}$-symmetry group of (22) (resp. (27)).

Proof. Immediate from 3.5 (ii) and 3.8 .

\subsection{Example Let}

$$
\Delta \equiv u_{t}+a(x, t) u_{x}+a_{0}(x, t, u)=0,(x, t \in \mathbb{R})
$$

then setting $z=\left(x, t, u, u_{x}, u_{t}\right)$ and using the notation of 3.8 we have $s=1, k_{s}=$ $5, \tilde{\Delta}(z)=\left(z_{1}, \ldots, z_{4}, z_{5}+a\left(z_{1}, z_{2}\right) z_{4}+a_{0}\left(z_{1}, z_{2}, z_{3}\right)\right), \tilde{\Delta}^{-1}(y)=\left(y_{1}, \ldots, y_{4}, y_{5}-\right.$ $\left.a\left(y_{1}, y_{2}\right) y_{4}-a_{0}\left(y_{1}, y_{2}, y_{3}\right)\right)$, and

$$
\begin{aligned}
\operatorname{pr}^{(1)} g_{\eta}(z)= & \left(\Xi_{\eta}\left(z_{1}, z_{2}\right), \Phi_{\eta}\left(z_{1}, z_{2}\right) z_{3}+\Psi_{\eta}\left(z_{1}, z_{2}\right), \sum_{j=3}^{5} b_{j}^{4}\left(\eta, z_{1}, z_{2}\right) z_{j}+\right. \\
& \left.b_{0}^{4}\left(\eta, z_{1}, z_{2}\right), \sum_{j=3}^{5} b_{j}^{5}\left(\eta, z_{1}, z_{2}\right) z_{j}+b_{0}^{5}\left(\eta, z_{1}, z_{2}\right)\right) \\
\Delta\left(\operatorname{pr}^{(1)} g_{\eta}(z)\right)= & \sum_{j=3}^{5} b_{j}^{5} z_{j}+b_{0}^{5}+a \cdot\left(\sum_{j=3}^{4} b_{j}^{4} z_{j}+b_{0}^{4}\right)+a_{0}
\end{aligned}
$$

Hence

$$
Q(y)=\frac{\partial}{\partial y_{5}}\left(f_{\eta} \circ \tilde{\Delta}^{-1}\right)(y)=b_{5}^{5}\left(\eta, y_{1}, y_{2}\right)+a\left(y_{1}, y_{2}\right) b_{2}^{4}\left(\eta, y_{1}, y_{2}\right) .
$$

Turning now to the distributional setting, we first note that the most general group actions applicable to distributions are those which are projectable and act linearly on the dependent variables, i.e. those which are of the form (23). If $u \in \mathcal{D}^{\prime}(\Omega)^{q}$ then the action of $g_{\eta}$ is defined by

$$
g_{\eta} u:=\Xi_{-\eta}^{*}\left(\Phi_{\eta} \cdot u+\Psi_{\eta}\right)
$$

where $\Xi_{-\eta}^{*}$ denotes (componentwise) distributional pullback, i.e.,

$$
\left.\left\langle f^{*}(u), \varphi\right\rangle=\left\langle u(y), \varphi\left(f^{-1}(y)\right)\left|\operatorname{det} D\left(f^{-1}\right)(y)\right|\right)\right\rangle \quad\left(u \in \mathcal{D}^{\prime}\left(\Omega^{\prime}\right), \varphi \in \mathcal{D}(\Omega)\right)
$$

for $f: \Omega \rightarrow \Omega^{\prime}$ a diffeomorphism. We will sometimes also write $u \circ f$ instead of $f^{*} u$.

3.12 Definition Suppose that (14) is linear and let $G$ be a local transformation group acting linearly on the dependent variables. $G$ is called a distributional (or $\mathcal{D}^{\prime}$-) symmetry group of (17) if it transforms distributional solutions of (1) into distributional solutions. 
$\overline{3.12}$ is the most general definition of distributional symmetry groups. More restrictive notions (as introduced e.g. in [5]) will be discussed in the following section.

3.13 Theorem Let $G$ be a symmetry group of the linear system (22) such that the assumptions of Theorem 3.8 are satisfied. Then $G$ is a distributional symmetry group of (22).

Proof. By 3.8 and (6), for any smooth $u: \Omega \rightarrow \mathbb{R}^{q}$ we have

$$
\Delta\left(x, \operatorname{pr}^{(n)}\left(g_{\eta} u\right)(x)\right)=Q\left(\eta, \Xi_{-\eta}(x)\right) \cdot \Delta\left(\Xi_{-\eta}(x), \operatorname{pr}^{(n)} u\left(\Xi_{-\eta}(x)\right)\right)
$$

Now suppose that $u \in \mathcal{D}^{\prime}(\Omega)^{q}$ is a solution to (11). Choose some $\phi \in \mathcal{D}\left(\mathbb{R}^{p}\right)$ with $\int \phi(x) d x=1$ and set $u_{\varepsilon}^{i}=u_{i} * \phi_{\varepsilon}, u_{\varepsilon}=\left(u_{\varepsilon}^{1}, \ldots, u_{\varepsilon}^{q}\right)$. Then $u_{\varepsilon}^{i}$ is smooth and converges to $u_{i}$ in $\mathcal{D}^{\prime}$ for $\varepsilon \rightarrow 0$. Since $g_{\eta}$ acts linearly on the dependent variables we also have that $\operatorname{pr}^{(n)}\left(g_{\eta} u_{\varepsilon}\right) \rightarrow \operatorname{pr}^{(n)}\left(g_{\eta} u\right)$ in $\mathcal{D}^{\prime}$. Hence

$$
\begin{aligned}
\Delta\left(x, \operatorname{pr}^{(n)}\left(g_{\eta} u\right)(x)\right) & =\lim _{\varepsilon \rightarrow 0} Q\left(\eta, \Xi_{-\eta}(x)\right) \cdot \Delta\left(\Xi_{-\eta}(x), \operatorname{pr}^{(n)} u_{\varepsilon}\left(\Xi_{-\eta}(x)\right)\right) \\
& =Q\left(\eta, \Xi_{-\eta}(x)\right) \cdot \Delta\left(\Xi_{-\eta}(x), \operatorname{pr}^{(n)} u\left(\Xi_{-\eta}(x)\right)\right)=0
\end{aligned}
$$

in $\mathcal{D}^{\prime}$, which concludes the proof.

\section{Infinitesimal criteria}

In this section we develop infinitesimal criteria for finding symmetries applicable to all three settings of interest (distributional, weak, Colombeau). The results introduced here also establish a direct connection of our approach to symmetries of distributional and weak solutions to that given in [5] (cf. also [3, $, 4,6]$ ).

In [5], systems of the form

$$
\left[\begin{array}{ccc}
L^{11} & \ldots & L^{1 q} \\
\ldots \ldots & \ldots & \ldots \\
L^{s 1} & \ldots & L^{s q}
\end{array}\right]\left[\begin{array}{c}
u_{1} \\
\ldots \\
u_{q}
\end{array}\right]=\left[\begin{array}{c}
F_{1} \\
\ldots \\
F_{s}
\end{array}\right], \text { or, for short } L(x, D) u=F
$$

where

$$
L^{i j}(x, D)=\sum_{|J|=0}^{n} a_{J}^{i j}(x) D^{J}, \quad a_{J}^{i j} \text { smooth }, \quad i=1, \ldots, s, j=1, \ldots, q
$$

( $n$ the order of $L$ ) and $F=x \mapsto F(x) \in \mathcal{D}^{\prime}(\Omega), \Omega \subseteq \mathbb{R}^{p}$, are examined. The form (28) of writing a system of linear PDEs (which, of course, is equivalent to (22)) provides the advantage of allowing to derive very concise forms of infinitesimal criteria for factorization properties, cf. (30) below.

In Berest's approach, symmetry groups of (28) are defined via factorization properties: Let $G=\left\{g_{\eta} \mid \eta \in\left(-\eta_{0}, \eta_{0}\right)\right\}$ be a projectable one parameter group acting linearly on the dependent variables. Thus $g_{\eta}(x, u)=(\Xi(\eta, x), \Phi(\eta, x, u))$ is of the form $(i=1, \ldots, p, k, l=1, \ldots, q)$

$$
\tilde{x}^{i}=\Xi^{i}(\eta, x), \tilde{u}^{k}=\Phi(\eta, x, u)=\sum_{l=1}^{q} \varphi^{k l}(\eta, x) u^{l}+\psi^{k}(\eta, x)
$$


$G$ is called a symmetry group of $(28)$ if there exists a smooth matrix valued map $(\eta, x) \rightarrow Q(\eta, x)$ such that (see [5], (1.6)):

$$
L(\tilde{x}, D)\left(g_{\eta} u\right)(\tilde{x})-F(\tilde{x})=Q(\eta, x)(L(x, D) u(x)-F(x)) .
$$

for all $u \in \mathcal{D}^{\prime}\left(\mathbb{R}^{p}\right)^{q}$ Note that this form corresponds exactly to (6) with $Q$ depending on $\eta$ and $x$ exclusively.

[5], (1.7) gives the following infinitesimal criterion for the validity of (29):

$$
[\xi D, L] u+L(\alpha(x) u+\beta(x))-\xi D F=\left.\frac{\partial Q}{\partial \eta}\right|_{\eta=0}(L u-F)
$$

where

$$
\xi=\left.\frac{\partial \Xi}{\partial \eta}\right|_{\eta=0}, \quad \xi D=\sum_{i=1}^{p} \xi_{i} \frac{\partial}{\partial x_{i}}, \quad \alpha=\left.\frac{\partial \varphi}{\partial \eta}\right|_{\eta=0}, \quad \beta=\left.\frac{\partial \psi}{\partial \eta}\right|_{\eta=0}
$$

The equivalence of (29) and (30) raises the question whether factorization properties for general systems (1) of differential equations can always be characterized by infinitesimal conditions similar to 30 . For $\Delta$ smooth, an affirmative answer is given by the following result, contained implicitly in [28] (cf. 4.2 (ii) below). Since its method of proof will form the basis for our generalizations to the $\mathcal{G}$ - resp. $\mathcal{D}^{\prime}$-settings we state it explicitly.

4.1 Proposition Let $G=\left\{g_{\eta} \mid \eta \in\left(-\eta_{0}, \eta_{0}\right)\right\}$ be a one parameter group with infinitesimal generator $\mathbf{v}$ acting on $\mathcal{M}$. Then the following are equivalent

(i) There exists a smooth mapping $Q: \mathcal{W} \rightarrow \mathbb{R}^{s^{2}}$ such that

$$
\Delta\left(\operatorname{pr}^{(n)} g_{\eta}(z)\right)=Q(\eta, z) \cdot \Delta(z) \quad((\eta, z) \in \mathcal{W})
$$

(ii) There exists a smooth mapping $\tilde{Q}: \mathcal{M}^{(n)} \rightarrow \mathbb{R}^{s^{2}}$ such that

$$
\operatorname{pr}^{(n)} \mathbf{v}(\Delta)(z)=\tilde{Q}(z) \cdot \Delta(z) \quad\left(z \in \mathcal{M}^{(n)}\right)
$$

Proof. (i) $\Rightarrow$ (ii): Noting that $\mathrm{pr}^{(n)} \mathbf{v}$ ( $\mathbf{v}$ the infinitesimal generator of $\eta \rightarrow g_{\eta}$ ) is a vectorfield on $\mathcal{M}^{(n)}$ whose flow is precisely $\mathrm{pr}^{(n)} g_{\eta}$, differentiation of (31) with respect to $\eta$ at $\eta=0$ gives (32) (with $\tilde{Q}(z)=\left.\frac{\partial}{\partial \eta}\right|_{\eta=0} Q(\eta, z)$ ).

(ii) $\Rightarrow$ (i): (32) yields the following linear ODE for $\Delta \circ \operatorname{pr}^{(n)} g_{\eta}$ :

$$
\begin{aligned}
\frac{\partial}{\partial \eta}\left(\Delta \circ \operatorname{pr}^{(n)} g_{\eta}(z)\right) & =\tilde{Q}\left(\operatorname{pr}^{(n)} g_{\eta}(z)\right) \cdot \Delta \circ \operatorname{pr}^{(n)} g_{\eta}(z) \\
\left.\Delta \circ \mathrm{pr}^{(n)} g_{\eta}(z)\right|_{\eta=0} & =\Delta(z)
\end{aligned}
$$

Let $Q(\eta, z)$ be a principal matrix solution to (33) (i.e. the $i$-th column of $Q$ is precisely the solution with initial value $e_{i}$ ). Then we immediately obtain the unique solution to (33) in the form

$$
\Delta \circ \operatorname{pr}^{(n)} g_{\eta}(z)=Q(\eta, z) \cdot \Delta(z)
$$

\subsection{Remarks}


(i) From the explicit formulae given in the above proof it follows that $Q$ depends exclusively on $z \in \mathcal{M}^{(k)}$ for $k<n$ (e.g. exclusively on $\left(z_{1}, \ldots, z_{p}\right)=$ $x)$ if and only if the same is true of $\tilde{Q}$ : indeed it suffices to note that by [28], (2.20) $\pi_{k}^{n} \circ \mathrm{pr}^{(n)} g_{\eta}=\operatorname{pr}^{(k)} g_{\eta}$ (where $\pi_{k}^{n}: \mathcal{M}^{(n)} \rightarrow \mathcal{M}^{(k)}$ is the natural projection).

(ii) By [28], eq. (2.26), for any nondegenerate system (11), (32) is equivalent to $\operatorname{pr}^{(n)} \mathbf{v}\left(\Delta_{i}\right)=0$ on the zero-set of $\Delta(1 \leq i \leq s)$ which in turn (by [28], Th. 2.71) is necessary and sufficient for $\mathbf{v}$ to generate a one-parameter symmetry group of (11). Thus (32) is precisely the infinitesimal version of the global factorization (5).

(iii) It follows immediately from the definition of $\mathrm{pr}^{(n)} g_{\eta}$ that (31) is equivalent with

$$
\begin{aligned}
& \Delta\left(\Xi_{\eta}(x, u(x)), \operatorname{pr}^{(n)} g_{\eta}(u)\left(\Xi_{\eta}(x, u(x))\right)\right) \\
& =Q\left(\eta, x, \operatorname{pr}^{(n)} u(x)\right) \cdot \Delta\left(x, \operatorname{pr}^{(n)} u(x)\right) \quad \forall u \in \mathcal{C}^{\infty}(\Omega)^{q} \forall x \in \Omega
\end{aligned}
$$

where $\Omega \subseteq \mathbb{R}^{p}$ runs through all open sets. A similar reformulation is valid for (32).

In the Colombeau setting the general form of (11) allows for $\Delta$ itself to be a generalized function. More precisely, we suppose that $\Delta \in \mathcal{G}_{\tau}\left(\mathcal{M}^{(n)}\right)^{s}$. Thereby, the admissible symmetry transformations themselves will become generalized functions, so-called (projectable) generalized group actions $g \in \tilde{\mathcal{G}}_{\tau}(\mathbb{R} \times \mathcal{M})^{p+q}$. Thus $g$ is supposed to satisfy $\mathcal{G}_{\tau}$-bounds with respect to the group parameter $\eta \in \mathbb{R}$ and $\mathcal{G}$-bounds with respect to $(x, u) \in \mathcal{M}$ ([21], Def. 4.8). Many of the infinitesimal methods of classical group analysis can be recovered in this setting. For a detailed analysis we refer to [21]. The proof of the analogue to 4.1 in the present situation requires the following auxiliary result on solutions of linear ODEs in $\mathcal{G}$ :

4.3 Lemma Let $A \in \tilde{\mathcal{G}}_{\tau}\left(\left(-\eta_{0}, \eta_{0}\right) \times \mathbb{R}^{p}\right)^{m^{2}}$ such that for all $K \subset \subset \mathbb{R}^{p}$,

$$
\sup _{x \in K} \int_{\mathbb{R}}\left\|A_{\varepsilon}(s, x)\right\| d s=O(|\log (\varepsilon)|)
$$

Then for each $u_{0} \in \mathcal{G}\left(\mathbb{R}^{p}\right)^{m}$ the initial value problem

$$
\begin{aligned}
\partial_{t} u(t, x) & =A(t, x) u(t, x) \\
u(0, x) & =u_{0}(x)
\end{aligned}
$$

has a unique solution $u$ in $\tilde{\mathcal{G}}_{\tau}\left(\left(-\eta_{0}, \eta_{0}\right) \times \mathbb{R}^{p}\right)^{m}$. Setting $U$ the matrix with columns the unique solutions with initial conditions $e_{i}(1 \leq i \leq m)$, the solution to (35) is given by $U(t, x) u_{0}(x)$. We call $U$ a principal matrix solution to (35).

Proof. We only sketch the argument (for details, cf. 14, 22, 23). Choosing representatives $\left(A_{\varepsilon}\right)_{\varepsilon}$ of $A$ and $\left(u_{0 \varepsilon}\right)_{\varepsilon}$ of $u_{0}$, by the corresponding result in the $\mathcal{C}^{\infty}$-setting we obtain representatives $\left(u_{\varepsilon}\right)_{\varepsilon},\left(U_{\varepsilon}\right)_{\varepsilon}$ satisfying the claimed properties for each fixed $\varepsilon$. Then

$$
\left\|u_{\varepsilon}(t, x)\right\| \leq\left\|u_{\varepsilon}(0, x)\right\|+\int_{0}^{t}\left\|A_{\varepsilon}(s, x)\right\|\left\|u_{\varepsilon}(s, x)\right\| d s, t \in\left(-\eta_{0}, \eta_{0}\right)
$$


Thus Gronwall's inequality and the supposed growth restriction on $A$ imply moderateness of the representatives and unique solvability.

Note that the corresponding statement for initial value problems in the sense of association (i.e. replacing $=$ by $\approx$ in $(35)$ ) is false since unique solvability of linear ODEs breaks down in that context. As an easy example take $u_{\varepsilon}(x)=\frac{1}{\varepsilon} \cos \left(\varepsilon^{2} x\right)$ $(x \in \mathbb{R})$. Then $u^{\prime} \approx 0$ but $u$ is not associated to any constant.

An element $u$ of $\mathcal{G}(\Omega)$ is called globally of $L^{\infty}$-log-type (cf. [14]) if it possesses a representative $\left(u_{\varepsilon}\right)_{\varepsilon}$ with $\sup _{x \in \Omega}\left|u_{\varepsilon}(x)\right|=O(|\log (\varepsilon)|)$. After these preparations we can state (for the notion of $\mathcal{G}$-n-completeness, see [21], Def. 4.15):

4.4 Proposition Let $\Delta \in \mathcal{G}_{\tau}\left(\mathcal{M}^{(n)}\right)^{s}$ and let $g$ be a $\mathcal{G}$-n-complete group action on $\mathcal{M}$. Consider the statements

(i) There exists $Q \in\left(\tilde{\mathcal{G}}_{\tau}(\mathcal{W})\right)^{s^{2}}$ with

$$
\Delta\left(\operatorname{pr}^{(n)} g_{\eta}(z)\right)=Q(\eta, z) \cdot \Delta(z) \text { in } \tilde{\mathcal{G}}_{\tau}(\mathcal{W})^{s}
$$

(ii) There exists $\tilde{Q} \in \mathcal{G}\left(\mathcal{M}^{(n)}\right)^{s^{2}}$

$$
\operatorname{pr}^{(n)} \mathbf{v}(\Delta)(z)=\tilde{Q}(z) \cdot \Delta(z) \text { in } \mathcal{G}\left(\mathcal{M}^{(n)}\right)^{s}
$$

Then (i) implies (ii). If $\tilde{Q} \circ \operatorname{pr}^{(n)} g_{\eta}$ is an element of $\tilde{\mathcal{G}}_{\tau}(\mathcal{W})^{s^{2}}$ satisfying the growth property given in 4.5 then (ii) implies (i). If $Q$ and $\tilde{Q}$ are supposed to be globally of $L^{\infty}$-log-type then (i) and (ii) are equivalent.

Proof. Using 4.3, the proof proceeds along the lines of 4.1 .

From the pointvalue characterization of Colombeau generalized functions given in 21] it follows that (36) is equivalent with

$$
\begin{aligned}
& \Delta\left(\Xi_{\eta}(x), \operatorname{pr}^{(n)} g_{\eta}(u)\left(\Xi_{\eta}(x)\right)\right) \\
& =Q\left(\eta, x, \operatorname{pr}^{(n)} u(x)\right) \cdot \Delta\left(x, \operatorname{pr}^{(n)} u(x)\right) \quad \text { in } \mathcal{G}(\Omega)^{s} \forall u \in \mathcal{G}(\Omega)^{q} \forall \Omega
\end{aligned}
$$

(cf. 21], Lemma 4.13 and Prop. 4.14).

4.5 Remark If in 4.3, $A \in \mathcal{C}^{\infty}\left(\left(-\eta_{0}, \eta_{0}\right) ; \mathcal{O}_{C}\left(\mathbb{R}^{n}\right)\right)^{m^{2}}$ and $u_{0} \in \mathcal{O}_{C}\left(\mathbb{R}^{n}\right)$, then the solution to (35), $U(t, x) u_{0}(x)$ belongs to $\mathcal{C}^{\infty}\left(\left(-\eta_{0}, \eta_{0}\right) ; \mathcal{O}_{C}\left(\mathbb{R}^{n}\right)\right)^{m}$. Thus, if we suppose $\Delta \in \mathcal{O}_{C}\left(\mathbb{R}^{N}\right)^{s}$ in 4.4 then we have $Q \in \mathcal{C}^{\infty}\left(\left(-\eta_{0}, \eta_{0}\right) ; \mathcal{O}_{C}\left(\mathbb{R}^{N}\right)\right)^{s^{2}}$, $\tilde{Q} \in \mathcal{O}_{C}\left(\mathbb{R}^{N}\right)^{s^{2}}$ and (i) and (ii) in 4.4 are equivalent

Turning now to the distributional setting, we first note the following result on linear ODEs in $\mathcal{D}^{\prime}$.

4.6 Lemma Let $A \in \mathcal{C}^{\infty}\left(\mathbb{R}^{p}\right)^{m^{2}}$ and let $a \in \mathcal{D}^{\prime}\left(\mathbb{R}^{p}\right)^{m}$. Then the initial value problem

$$
\begin{aligned}
\partial_{t} u & =A \cdot u & & \text { in } \mathcal{C}^{\infty}\left(\mathbb{R}, \mathcal{D}^{\prime}\left(\mathbb{R}^{p}\right)^{m}\right) \\
u(0, .) & =a & & \text { in } \mathcal{D}^{\prime}\left(\mathbb{R}^{p}\right)^{m}
\end{aligned}
$$

has the unique solution $Q \cdot a$ where $Q$ is the smooth principal matrix solution of (39). 
Proof. That $Q \cdot a$ is a solution follows easily by regularizing the initial data via convolution with a standard mollifier and then taking the distributional limit of the solutions to the resulting smooth problems. Uniqueness follows from uniqueness of the corresponding smooth initial value problem by observing that for any solution $u \in \mathcal{C}^{\infty}\left(\mathbb{R}, \mathcal{D}^{\prime}\left(\mathbb{R}^{p}\right)^{m}\right)$ of $(39)$ with $a=0$ we have $\partial_{t}\left(Q^{-1} u\right)=0$. But then $u=Q \cdot c$ with $c$ a constant vector which is necessarily 0 .

Let us suppose that $\Delta$ is of the form (22) with $a_{0}^{i} \in \mathcal{D}^{\prime}\left(\mathbb{R}^{p}\right)$ and $a_{k}^{i} \in \mathcal{C}^{\infty}\left(\mathbb{R}^{p}\right)$ $(1 \leq i \leq s, p+1 \leq k \leq N)$. This is the most general form of differential operators applicable to elements $u$ of $\mathcal{D}^{\prime}\left(\mathbb{R}^{p}\right)$. Moreover, we suppose that the group action $g_{\eta}$ is of the form (23) (also the most general action applicable to distributions). We consider $\Delta$ as an element of $\mathcal{D}^{\prime}\left(\mathbb{R}^{N}\right)$ by embedding $a_{0}$ as $a_{0} \otimes 1_{N-p}$ into $\mathcal{D}^{\prime}\left(\mathbb{R}^{N}\right)$. For the following result, to simplify notations we suppose that $\mathcal{M}=\mathbb{R}^{p} \times \mathbb{R}^{q}$ and that $g$ is defined on all of $\mathcal{M}$.

4.7 Proposition Under the assumptions formulated before 4.6, the following are equivalent

(i) There exists a smooth mapping $Q: \mathbb{R} \times \mathbb{R}^{p} \rightarrow \mathbb{R}^{s^{2}}$ such that

$$
\left(\operatorname{pr}^{(n)} g_{\eta}\right)^{*} \Delta=Q(\eta, .) \cdot \Delta \text { in } \mathcal{C}^{\infty}\left(\mathbb{R}, \mathcal{D}^{\prime}\left(\mathcal{M}^{(n)}\right)^{s}\right)
$$

(ii) There exists a smooth mapping $\tilde{Q}: \mathbb{R}^{p} \rightarrow \mathbb{R}^{s^{2}}$ such that

$$
\operatorname{pr}^{(n)} \mathbf{v}(\Delta)=\tilde{Q} \cdot \Delta \text { in } \mathcal{D}^{\prime}\left(\mathcal{M}^{(n)}\right)^{s}
$$

(iii) There exists a smooth mapping $Q: \mathbb{R} \times \mathbb{R}^{p} \rightarrow \mathbb{R}^{s^{2}}$ such that

$$
\Delta\left(\Xi_{\eta}(.), \operatorname{pr}^{(n)}\left(g_{\eta} u\right)\right)=Q(\eta, .) \cdot \Delta\left(., \operatorname{pr}^{(n)} u(.)\right) \text { in } \mathcal{C}^{\infty}\left(\mathbb{R}, \mathcal{D}^{\prime}\left(\mathbb{R}^{p}\right)^{s}\right)
$$

for all $u \in \mathcal{D}^{\prime}\left(\mathbb{R}^{p}\right)$.

Proof. (i) $\Leftrightarrow$ (ii): Using 4.6, the proof is again identical to that of 4.1 (for the distributional identity $\frac{\partial}{\partial \eta}\left(\left(\mathrm{pr}^{(n)} g_{\eta}\right)^{*} \Delta\right)=\operatorname{pr}^{(n)} \mathbf{v}(\Delta)$ used in the argument, see e.g., [24, Th. 3.7).

(i) $\Leftrightarrow$ (iii): With the notations introduced in (22), (25), (26) and $z=\left(x_{1}, \ldots, x_{p}\right.$, $\left.z_{p+1}, \ldots, z_{N}\right)$ we have

$$
\begin{aligned}
\Delta_{i}\left(\operatorname{pr}^{(n)} g_{\eta}(z)\right)= & \sum_{j=p+1}^{N} a_{j}^{i}(\Xi(\eta, x))\left(\sum_{l=p+1}^{N} b_{l}^{j}(\eta, x) z_{l}+b_{0}^{j}(\eta, x)\right)+ \\
& a_{0}^{i}(\Xi(\eta, x)) \otimes 1_{N-p} \quad(i=1, \ldots, s)
\end{aligned}
$$

It follows that (40) can be written in the form

$$
\begin{aligned}
& \sum_{l=p+1}^{N}\left(\sum_{j=p+1}^{N} a_{j}^{i}(\Xi(\eta, x)) b_{l}^{j}(\eta, x)\right) z_{l}+\sum_{j=p+1}^{N} a_{j}^{i}(\Xi(\eta, x)) b_{0}^{j}(\eta, x)+ \\
& a_{0}^{i}(\Xi(\eta, x)) \otimes 1_{N-p}=\sum_{m=1}^{s} Q_{i m}(x)\left(\sum_{k=p+1}^{N} a_{k}^{m}(x) z_{k}+a_{0}^{m}(x) \otimes 1_{N-p}\right)
\end{aligned}
$$


Hence, introducing suitable smooth functions $\tilde{a}_{k}^{i}$ and distributions $\tilde{a}_{0}^{i}(k=p+$ $1, \ldots, N, i=1, \ldots, s)$, the proof reduces to establishing the equivalence of

$$
\begin{aligned}
& \left\langle\sum_{k=p+1}^{N} \tilde{a}_{k}^{i}(\eta, x) z_{k}+\tilde{a}_{0}^{i}(x) \otimes 1_{N-p}, \varphi_{1}\left(x_{1}\right) \ldots \varphi_{p}\left(x_{p}\right) \psi_{p+1}\left(z_{p+1}\right) \ldots \psi_{N}\left(z_{N}\right)\right\rangle \\
& =0, \quad \varphi_{m}, \psi_{n} \in \mathcal{D}(\mathbb{R}), m=1, \ldots, p, n=p+1, \ldots, N
\end{aligned}
$$

and

$$
\begin{aligned}
& \left\langle\sum_{k=p+1}^{N} \tilde{a}_{k}^{i}(\eta, x) z_{k}(x)+\tilde{a}_{0}^{i}(x) \otimes 1_{N-p}, \varphi_{1}\left(x_{1}\right) \ldots \varphi_{p}\left(x_{p}\right)\right\rangle=0, \\
& \varphi_{m} \in \mathcal{D}(\mathbb{R}), m=1, \ldots, p, z_{k} \in \mathcal{C}^{\infty}\left(\mathbb{R}^{p}\right), k=p+1, \ldots, N
\end{aligned}
$$

This last assertion naturally splits into a (distributional) invariance property of $\tilde{a}_{0}^{i} \otimes 1_{N-p}$ resp. $\tilde{a}_{0}^{i}$ and a smooth part, both of which are easily seen to be equivalent.

Thus (29) corresponds precisely to (42). In particular, the specific form of the infinitesimal criterion for the validity of (29) follows from an explicit calculation of $\operatorname{pr}^{(n)} \mathbf{v}(\Delta)$ in the notation (due to Berest) introduced at the beginning of this section. In fact, we have

$$
\mathbf{v}=\sum_{i=1}^{p} \xi^{i}(x) \partial_{x_{i}}+\sum_{k=1}^{q} \underbrace{\left(\sum_{l=1}^{q} \alpha^{k l}(x) u^{l}+\beta^{k}(x)\right)}_{=: \Phi_{k}(x, u)} \partial_{u_{k}}
$$

Then by [28], Th. 2.36, $\operatorname{pr}^{(n)} \mathbf{v}=\sum_{i=1}^{p} \xi^{i} \partial_{x_{i}}+\sum_{k=1}^{q} \sum_{|J|=0}^{n} \Phi_{k}^{J}\left(x, u^{(n)}\right) \partial_{u_{J}^{k}}$, where

$$
\Phi_{k}^{J}\left(x, u^{(n)}\right)=D_{J}\left(\Phi_{k}-\sum_{i=1}^{p} \xi_{i} \frac{\partial u^{k}}{\partial x^{i}}\right)+\sum_{i=1}^{p} \xi_{i} u_{J, i}^{k}
$$

$\left(u_{J, i}^{k}=\frac{\partial u_{j}^{k}}{\partial x_{i}}\right)$. Thus the $r$-th component of $\operatorname{pr}^{(n)} \mathbf{v}(\Delta)=\operatorname{pr}^{(n)} \mathbf{v}(L(x, D) u-F)$ is calculated as follows:

$$
\begin{aligned}
& \operatorname{pr}^{(n)} \mathbf{v}\left(\sum_{k=1}^{q} \sum_{|J|=0}^{n} a_{J}^{r k} u_{J}^{k}\right)-\operatorname{pr}^{(n)} \mathbf{v}\left(F_{r}\right) \\
& =\sum_{k=1}^{q} \sum_{|J|=0}^{n} \sum_{i=1}^{p} \xi_{i} \partial_{i}\left(a_{J}^{r k}\right) u_{J}^{k}+\sum_{k=1}^{q} \sum_{|J|=0}^{n} \Phi_{k}^{J} a_{J}^{r k}-\sum_{i=1}^{p} \xi_{i} \partial_{i} F_{r} \\
& =\sum_{k=1}^{q} \sum_{|J|=0}^{n} \sum_{i=1}^{p} \xi_{i} \partial_{i}\left(a_{J}^{r k}\right) u_{J}^{k}-\sum_{k=1}^{q} \sum_{|J|=0}^{n} \sum_{i=1}^{p} a_{J}^{r k} \partial^{J} \xi_{i} \partial_{i} u^{k} \\
& +\sum_{k=1}^{q} \sum_{|J|=0}^{n} \sum_{l=1}^{q} a_{J}^{r k} \partial^{J} \alpha^{k l} u^{l}+\sum_{k=1}^{q} \sum_{|J|=0}^{n} \sum_{l=1}^{q} \alpha^{k l} a_{J}^{r k} \partial^{J} u^{l} \\
& +\sum_{k=1}^{q} \sum_{|J|=0}^{n} a_{J}^{r k} \partial^{J} \beta^{k}-\sum_{i=1}^{p} \xi_{i} \partial_{i} F_{r} \\
& =([\xi D, L] u+L(x, D)(\alpha u+\beta)-\xi D F)_{r}
\end{aligned}
$$


This calculation, combined with 4.7 and (42) provides a rigorous proof of the equivalence of (29) and (30). Moreover, 4.1 allows to derive infinitesimal criteria for factorization properties even for systems that are not necessarily linear and thereby to obtain workable criteria for finding symmetries of weak or Colombeau solutions of such systems. For example, let us consider the semilinear system

$$
L(x, D) u=F(u)
$$

where we shall suppose $F \in \mathcal{O}_{M}\left(\mathbb{R}^{q}\right)$ (to allow for an insertion of Colombeau functions, note however that 4.8 below does not use this assumption). Furthermore, let us assume that the group action $g_{\eta}$ is of the form (23). Then we have

4.8 Proposition Under the above assumptions, the following are equivalent:

(i) There exists a smooth mapping $Q: \mathcal{W} \rightarrow \mathbb{R}^{s^{2}}$ such that for all $u \in \mathcal{C}^{\infty}(\Omega)$ $\left(\Omega \subseteq \mathbb{R}^{p}\right)$ and all $x \in \Omega$

$$
L(\tilde{x}, D)\left(g_{\eta} u\right)(\tilde{x})-F\left(g_{\eta} u(\tilde{x})\right)=Q(\eta, x)(L(x, D) u(x)+F(u(x)))
$$

(ii) There exists a smooth mapping $\tilde{Q}: \mathcal{M}^{(n)} \rightarrow \mathbb{R}^{s^{2}}$ such that for all $u \in$ $\mathcal{C}^{\infty}(\Omega)\left(\Omega \subseteq \mathbb{R}^{p}\right)$ and all $x \in \Omega$

$$
\begin{aligned}
& {[\xi D, L] u(x)+L(x, D)(\alpha(x) u(x)+\beta(x))-J(F)(u(x))} \\
& (\alpha(x) \cdot u(x)+\beta(x))=\tilde{Q}(x)(L(x, D) u(x)-F(u(x)))
\end{aligned}
$$

(with $J(F)$ the Jacobian of $F$ ).

Proof. By 4.1 and 4.2 (iii) it suffices to calculate $\operatorname{pr}^{(n)} \mathbf{v}(\Delta)$ for $\Delta=L(x, D) u-$ $F(u)$ as above. Noting that $\operatorname{pr}^{(n)} \mathbf{v}(F)=J(F)(u) \cdot(\alpha \cdot u+\beta)$, the result follows exactly as in the above calculation.

In [5], it was shown that a certain splitting of (30) is advantageous for establishing a connection between determining symmetry groups of PDEs and group invariance of the solutions themselves, in particular with a view to determining group invariant fundamental solutions of linear systems. In our more general setup, we first note that (using obvious abbreviations) setting $K=\xi D-\alpha$ and $h=\alpha-\left.\frac{\partial}{\partial \eta}\right|_{\eta=0}$, (30) is equivalent to (46) as well as to (47). Also, (45) is equivalent to 48$)$ :

$$
\begin{aligned}
& (-[L, K]+h L) u-(K+h) F+L \beta=0 \\
& {[L, K]=h L,(K+H) F=L \beta} \\
& (-[L, K]+h L) u-J(F)(u) \alpha u+\left.\frac{\partial}{\partial \eta}\right|_{\eta=0} Q F(u)-J(F)(u) \beta=0
\end{aligned}
$$

We first note the following immediate consequence of (30):

4.9 Proposition Let $\eta \rightarrow g_{\eta}$ be a one-parameter symmetry group of the form (23) of the homogeneous system $L(x, D) u=0$ satisfying (29). If $u \in \mathcal{G}(\Omega)^{q}$ is a solution, resp. associated solution resp. strongly associated solution then so is Ku. 
4.10 Definition Let $\eta \rightarrow g_{\eta}$ be a slowly increasing one-parameter group. We say that $u \in \mathcal{G}(\Omega)^{q}$ is $\mathcal{G}$-invariant under $g_{\eta}$, $\approx$-invariant, or $\stackrel{k}{\approx}$-invariant, respectively, if $g_{\eta} u=u, g_{\eta} u \approx u$, or $g_{\eta} u \stackrel{k}{\approx} u$ for all $\eta$. If $g_{\eta}$ is of the form (2O) and $u \in \mathcal{D}^{\prime}(\Omega)^{q}$ then $u$ is called $\mathcal{D}^{\prime}$-invariant under $g_{\eta}$ if $g_{\eta} u=u$ in $\mathcal{D}^{\prime}$.

4.11 Proposition Let $\eta \rightarrow g_{\eta}$ be of the form (23).

(i) Let $u \in\left(\mathcal{C}^{\infty}\right)^{q}$ resp. $u \in \mathcal{G}^{q}$ resp. $u \in\left(\mathcal{D}^{\prime}\right)^{q} . A$ necessary and sufficient condition for $u$ to be invariant resp. $\mathcal{G}$-invariant resp. $\mathcal{D}^{\prime}$-invariant under $g_{\eta}$ is that $K u$ equals $\beta$ in $\mathcal{C}^{\infty}$ resp. in $\mathcal{G}$ resp. in $\mathcal{D}^{\prime}$.

(ii) Then $u$ is $\approx$-invariant, resp. $\stackrel{k}{\approx}$-invariant, if $K u-\beta \approx 0$, resp. $K u-\beta \stackrel{k}{\approx} 0$ $\left(k \in \mathbb{N}_{0}\right)$.

Proof. (i) We first note that in each of the possible settings we have

$$
\left.\frac{\partial}{\partial \eta}\right|_{0}\left(g_{\eta} u\right)=-K u+\beta
$$

which is immediate from the chain rule. Thus the conditions are necessary. Conversely, for fixed $u$ set

$$
f(\eta, x)=\Xi_{\eta}^{*} u(x)-\phi(\eta, x) u(x)-\psi(\eta, x)
$$

Then $f^{\prime}(\eta, x)=\Xi_{\eta}^{*} \alpha \cdot f(\eta, x)$ and $f(0, x)=0$. Thus the claim follows from unique solvability of linear ODEs in each of the respective settings (cf. 4.3 with $A$ smooth (hence automatically satisfying the necessary growth restrictions) and 4.6.).

(ii) Setting $r_{\varepsilon}(\eta,)=.\Xi_{\eta}^{*}\left(K u_{\varepsilon}-\beta\right)$ we have

$$
\begin{aligned}
& f_{\varepsilon}^{\prime}(\eta, x)=\Xi_{\eta}^{*} \alpha \cdot f_{\varepsilon}(\eta, x)+r_{\varepsilon}(\eta, x) \\
& f_{\varepsilon}(0, x)=0
\end{aligned}
$$

Also, $r_{\varepsilon}(\eta,.) \approx 0$ resp. $\stackrel{k}{\approx} 0$ for all $\eta$ follows from our assumption (by substituting for $\Xi_{\eta}(x)$ in the respective integrals). With $U(\eta, x)$ the principal matrix solution to the corresponding homogeneous system we obtain the solution to this initial value problem in the form

$$
f_{\varepsilon}(\eta, x)=U(\eta, x) \int_{0}^{\eta} U\left(\eta^{\prime}, x\right)^{-1} r_{\varepsilon}(\eta, x) d \eta^{\prime}
$$

Thus for $\varphi \in \mathcal{D}$, resp. $\varphi \in B \subseteq \mathcal{C}_{c}^{\infty}, B$ bounded in $\mathcal{C}_{c}^{k}$, we have

$$
\int f_{\varepsilon}(\eta, x) \varphi(x) d x=\int r_{\varepsilon}(\eta, x)\left(U(\eta, x) \int_{0}^{\eta} U\left(\eta^{\prime}, x\right)^{-1} d \eta^{\prime} \varphi(x)\right) d x \rightarrow 0
$$

as $\varepsilon \rightarrow 0$, resp. this limit is uniform for $\varphi \in B$. It follows that $f_{\varepsilon}(\eta,.) \approx 0$, resp. $f_{\varepsilon}(\eta,.) \stackrel{k}{\approx} 0$ i.e., that $g_{\eta} u_{\varepsilon} \approx u_{\varepsilon}$, resp. $g_{\eta} u_{\varepsilon} \stackrel{k}{\approx} u_{\varepsilon}$.

Note that the converse assertion in (ii) does not follow since $g_{\eta} u-u \approx 0$ resp. $\stackrel{k}{\approx} 0$ for all $\eta$ does not imply any information on $\left.\frac{d}{d \eta}\right|_{0} g_{\eta} u=-K u_{\varepsilon}+\beta$. 
4.12 Example We derive infinitesimal criteria for symmetries (16) of the quasilinear system (15) whose infinitesimal generators we write as

$$
\xi(x, t) \partial_{x}+\tau(x, t) \partial_{t}+\psi(x, t, u) \partial_{u}
$$

Applying $\left.\frac{\partial}{\partial \eta}\right|_{\eta=0}$ to $\Phi_{\eta}^{1} J \Xi_{\eta}=\left[\begin{array}{ll}\Phi_{\eta x} & \Phi_{\eta t}\end{array}\right]+\Phi_{\eta u}\left[\begin{array}{ll}u_{x} & u_{t}\end{array}\right]$, which is just the short form of (17), we obtain

$$
\left.\frac{\partial}{\partial \eta}\right|_{\eta=0} \Phi_{\eta}^{1}+\left.\left[\begin{array}{ll}
u_{x} & u_{t}
\end{array}\right] \frac{\partial}{\partial \eta}\right|_{\eta=0} J \Xi_{\eta}=\left[\begin{array}{ll}
\psi_{x} & \psi_{t}
\end{array}\right]+\psi_{u}\left[\begin{array}{ll}
u_{x} & u_{t}
\end{array}\right]
$$

Thus,

$$
\begin{aligned}
& \left.\frac{\partial}{\partial \eta}\right|_{\eta=0} \Phi_{\eta}^{1}\left[\begin{array}{l}
1 \\
0
\end{array}\right]=\psi_{x}+\psi_{u} u_{x}-\xi_{x} u_{x}-\tau_{x} u_{t} \\
& \left.\frac{\partial}{\partial \eta}\right|_{\eta=0} \Phi_{\eta}^{1}\left[\begin{array}{l}
0 \\
1
\end{array}\right]=\psi_{t}+\psi_{u} u_{t}-\xi_{t} u_{x}-\tau_{t} u_{t}
\end{aligned}
$$

Hence by (18) we get the following expression for $\left.\frac{\partial}{\partial \eta}\right|_{\eta=0} \tilde{\Delta}_{\eta}$ :

$$
\begin{gathered}
\left.\frac{\partial}{\partial \eta}\right|_{\eta=0} \Phi_{\eta}^{1}\left[\begin{array}{l}
0 \\
1
\end{array}\right]+\left.\frac{\partial}{\partial \eta}\right|_{\eta=0}\left(A\left(\Phi_{\eta}\right)\right)\left[u_{x} u_{t}\right]\left[\begin{array}{l}
1 \\
0
\end{array}\right]+\left.A(u) \frac{\partial}{\partial \eta}\right|_{\eta=0} \Phi_{\eta}^{1}\left[\begin{array}{l}
1 \\
0
\end{array}\right]= \\
{\left[\psi_{t}\right]_{1}^{s}+\left[\psi_{u}\right]_{s}^{s}\left[u_{t}\right]_{1}^{s}-\xi_{t}\left[u_{x}\right]_{1}^{s}-\tau_{t}\left[u_{t}\right]_{1}^{s}+\sum_{i} \psi^{i}\left[\frac{\partial A}{\partial u^{i}}\right]_{s}^{s}\left[u_{x}\right]_{1}^{s}+} \\
{[A(u)]_{s}^{s}\left(\left[\psi_{x}\right]_{1}^{s}+\left[\psi_{u}\right]_{s}^{s}\left[u_{x}\right]_{1}^{s}-\xi_{x}\left[u_{x}\right]_{1}^{s}-\tau_{x}\left[u_{t}\right]_{1}^{s}\right)=\left(\psi_{t}+A(u) \psi_{x}\right)+} \\
\left(\psi_{u}-\tau_{t}-\tau_{x} A(u)\right) u_{t}+\left(A(u) \psi_{u}-\xi_{t} I_{s}-\xi_{x} A(u)+\sum_{i} \psi^{i} \frac{\partial A}{\partial u^{i}}\right) u_{x}
\end{gathered}
$$

(where $[a]_{c}^{b}$ is a reminder that $a$ is $b \times c$ matrix). Note that in this expression the coefficient of $u_{t}$ equals $\left.\frac{\partial}{\partial \eta}\right|_{\eta=0} Q_{\eta}$.

Thus the determining system $\left(\left.\frac{\partial}{\partial \eta}\right|_{\eta=0} \tilde{\Delta}\right)_{\eta}=\left.\frac{\partial}{\partial \eta}\right|_{\eta=0} Q_{\eta} \cdot \Delta$ for an infinitesimal projectable symmetry reads

$$
\begin{aligned}
\psi_{t}+A(u) \psi_{x} & =0 \\
A(u) \psi_{u}-\xi_{x} A(u)-\xi_{t} \mathrm{I}_{s}+\sum_{i} \psi^{i} \frac{\partial A}{\partial u^{i}} & =\left(\psi_{u}-\tau_{t} \mathrm{I}_{s}-\tau_{x} A(u)\right) A(u)
\end{aligned}
$$

or

$$
\begin{aligned}
\psi_{t}+A(u) \psi_{x} & =0 \\
{\left[A(u), \psi_{u}\right]+\sum_{i} \psi^{i} \frac{\partial A}{\partial u^{i}} } & =\left(\xi_{t} \mathrm{I}_{s}-\tau_{t} A(u)\right)+\left(\xi_{x} \mathrm{I}_{s}-\tau_{x} A(u)\right) A(u)
\end{aligned}
$$

4.13 Example We continue to analyze the last determining system in the case of a strictly hyperbolic conservation law

$$
\begin{aligned}
u_{t}+f(u, v)_{x} & =0 \\
v_{t}+g(u, v)_{x} & =0 .
\end{aligned}
$$


corresponding to (15) with

$$
A=\left[\begin{array}{ll}
f_{u} & f_{v} \\
g_{u} & g_{v}
\end{array}\right]
$$

with the characteristic values $\lambda_{1}(u, v)<\lambda_{2}(u, v),(u, v) \in H$, where $H$ is open in $\mathbb{R}^{2}$ (cf. [8, 17, 18]; for generalized solutions, see [26).

We denote by $r_{i}=\left(\begin{array}{c}r_{i}^{1} \\ r_{i}^{2}\end{array}\right), \quad \ell_{i}=\left(\begin{array}{c}\ell_{i}^{1} \\ \ell_{i}^{2}\end{array}\right) \in\left(\mathcal{C}^{\infty}(H)\right)^{2 \times 1}$ the characteristic vectors: $A r_{i}=\lambda_{i} r_{i}, \quad \ell_{i}^{T} A=\lambda_{i} \ell_{i}^{T}, i=1,2$.

We will calculate the coefficients of an infinitesimal symmetry $\mathbf{v}=\xi(x, t) \partial_{x}+$ $\tau(x, t) \partial_{t}+\phi(x, t, u, v) \partial_{u}+\psi(x, t, u, v) \partial_{v}$ for (49), (50). By 4.12 we have

$$
\begin{aligned}
{\left[\begin{array}{l}
\phi_{t} \\
\psi_{t}
\end{array}\right]+A\left[\begin{array}{l}
\phi_{x} \\
\psi_{x}
\end{array}\right] } & =0 \\
{[A, B]+\phi A_{u}+\psi A_{v} } & =\left(\xi \mathrm{I}_{2}-\tau A\right)_{t}+\left(\xi \mathrm{I}_{2}-\tau A\right)_{x} A
\end{aligned}
$$

where $B=\left[\begin{array}{ll}\phi_{u} & \phi_{v} \\ \psi_{u} & \psi_{v}\end{array}\right]$.

Differentiating (51) with respect to $u$ and $v$ we have

$$
\begin{aligned}
& \left(B_{t}+\phi_{x} A_{u}+\psi_{x} A_{v}\right)\left[\begin{array}{l}
1 \\
0
\end{array}\right]+A\left[\begin{array}{l}
\phi_{x u} \\
\psi_{x u}
\end{array}\right]=0 \\
& \left(B_{t}+\phi_{x} A_{u}+\psi_{x} A_{v}\right)\left[\begin{array}{l}
0 \\
1
\end{array}\right]+A\left[\begin{array}{l}
\phi_{x v} \\
\psi_{x v}
\end{array}\right]=0
\end{aligned}
$$

Putting both together we obtain the matrix equation

$$
B_{t}+A B_{x}+\phi_{x} A_{u}+\psi_{x} A_{v}=0
$$

Taking the derivative of (52) with respect to $x$ and subtracting from (53) we arrive at

$$
B_{t}+B_{x} A+\left(\xi \mathrm{I}_{2}-\tau A\right)_{x t}+\left(\xi \mathrm{I}_{2}-\tau A\right)_{x x} A=0
$$

or in shorter form

$$
M_{t}+M_{x} A=0 \quad \text { where } \quad M=B+\left(\xi_{x} \mathrm{I}_{2}-\tau_{x} A\right)
$$

Thus we obtain the system (51), (54) which, while not equivalent to (51), (52) (due to the differentiations used in deriving it), considerably facilitates the determination of infinitesimal symmetries.

By 35], Section 16, we have

$$
\begin{gathered}
{\left[\begin{array}{l}
\phi(x, t, u, v) \\
\psi(x, t, u, v)
\end{array}\right]=\sum_{i=1}^{2} \alpha_{i}\left(x-t \lambda_{i}(u, v), u, v\right) r_{i}(u, v), \quad \alpha_{i} \in \mathcal{C}^{\infty}(\mathbb{R} \times H)} \\
M(x, t, u, v)=\sum_{i=1}^{2} \beta_{i}\left(x-t \lambda_{i}(u, v), u, v\right) \ell_{i}^{T}(u, v) \\
\beta_{i} \in\left(\mathcal{C}^{\infty}(\mathbb{R} \times H)\right)^{2 \times 1},(x, t, u, v) \in \mathbb{R}^{2} \times H
\end{gathered}
$$

In order to determine $\xi$ and $\tau$ as well as $\alpha_{i}, \beta_{i}=\left[\begin{array}{c}\beta_{i}^{1} \\ \beta_{i}^{2}\end{array}\right], i=1,2$, we use $M=B+\xi_{x} I_{2}-\tau_{x} A$ :

$$
\sum_{i=1}^{2} \beta_{i}^{1} \ell_{i}^{1}=\sum_{i=1}^{2}\left(\alpha_{i} r_{i}^{1}\right)_{u}+\xi_{x}-\tau_{x} f_{u} ; \sum_{i=1}^{2} \beta_{i}^{1} \ell_{i}^{2}=\sum_{i=1}^{2}\left(\alpha_{i} r_{i}^{1}\right)_{v}-\tau_{x} f_{v}
$$




$$
\sum_{i=1}^{2} \beta_{i}^{2} \ell_{i}^{1}=\sum_{i=1}^{2}\left(\alpha_{i} r_{i}^{2}\right)_{u}-\tau_{x} g_{u} ; \sum_{i=1}^{2} \beta_{i}^{2} \ell_{i}^{2}=\sum_{i=1}^{2}\left(\alpha_{i} r_{i}^{2}\right)_{v}+\xi_{x}-\tau_{x} g_{v}
$$

By 4.1 resp. 4.4, solutions of this system in $\mathcal{C}^{\infty}$ resp. $\mathcal{G}$ determine infinitesimal symmetries in each of the respective settings.

Note that the above considerations also hold for systems of order $s>2$ even in non-conservative form with the additional assumption that the coefficients of $A$ do not depend on $x$ and $t$.

\section{References}

[1] J. Aragona, H. A. Biagioni, Intrinsic definition of the Colombeau algebra of generalized functions, Analysis Mathematica, 17 (1991), 75-132.

[2] J. M. BALL, Global invertibility of Sobolev functions and the interpenetration of matter, Proc. Royal Soc. Edinburgh A 88 (1981), 315-328.

[3] Yu. Yu. Berest, Construction of fundamental solutions for Huygen's equations as invariant solutions, Soviet Math. Dokl., Vol. 43, No. 2, (1991) 496-499.

[4] Yu. Yu. Berest, Weak invariants of local groups of transformations, Diff. Equ., 29, No. 10 (1993), 1561-1567.

[5] Yu. Yu. Berest, Group analysis of linear differential equations in distributions and the construction of fundamental solutions, Diff. Equ., 29, No. 11 (1993), 1700-1711.

[6] Yu. Yu. Berest, N. H. Ibragimov, Group theoretic determination of fundamental solutions, Lie Groups Appl. 1, No. 2, (1994), 65-80.

[7] H. A. Biagioni, A Nonlinear Theory of Generalized Functions, Springer-Verlag, BerlinHeidelberg-New York, 1990.

[8] A. Bressan, Hyperbolic systems of conservation laws, Rev. Math. Comp. 12, 1 (1999), $135-200$

[9] J. F. Colombeau, New Generalized Functions and Multiplication of Distributions, North Holland, Amsterdam 1984.

[10] J. F. Colombeau, Elementary Introduction to New Generalized Functions, North Holland, Amsterdam, 1985.

[11] J. F.Colombeau, Multiplication of Distributions. A tool in mathematics, numerical engineering and theoretical physics, Lecture Notes in Mathematics 1532, Springer, Berlin 1992.

[12] M. Grosser, E. Farkas, M. Kunzinger, R. Steinbauer On the foundations of nonlinear generalized functions I, II. Mem. Am. Math. Soc. 153, No. 729, 2001.

[13] M. Grosser, M. Kunzinger, R. Steinbauer, J. Vickers A global theory of algebras of generalized functions. Adv. Math., to appear.

[14] R. Hermann, M. Oberguggenberger, Ordinary differential equations and generalized functions, in: M. Grosser, G. Hörmann, M. Kunzinger, M. Oberguggenberger (Eds.), Nonlinear Theories of Generalized Functions, CRC Research Notes in Mathematics 401 (1999), 85-98.

[15] N. H. Ibragimov, Group theoretical treatment of fundamental solutions, Physics on manifolds (Paris, 1992), 161-175, in M. Flato, R. Kerner and A. Lichnerowicz (Eds.), Math. Phys. Stud., 15, Kluwer Acad. Publ., Dordrecht, 1994.

[16] N.H. Ibragimov (Ed.), CRC Handbook of Lie Group Analysis of Differential Equations, Vol. 1-3, CRC Press, Florida, 1994 - 1996.

[17] B. L. Keyfitz, H.C.Kranzer, Spaces of weighted measures for conservation laws with singular shock solutions, J.Diff. Eq. 118 (1995), 420-451.

[18] B. L. Keyfitz, H. C. Kranzer, Existence and uniqueness of entropy solutions for the Riemann problem for hyperbolic systems of two nonlinear conservation laws, J. Diff. Equ. 27 (1978), 444-476. 
[19] M. Kunzinger, Lie Transformation Groups in Colombeau Algebras, PhD Thesis, University of Vienna, 1996.

[20] M. Kunzinger, M. Oberguggenberger, Symmetries of Differential Equations in Colombeau Algebras, in: N. H. Ibragimov, F. M. Mahomed (Eds.), Modern Group Analysis VI, New Age Int. Publ., New Delhi, 9-20, (1997).

[21] M. Kunzinger, M. Oberguggenberger, Group analysis of differential equations and generalized functions, SIAM J. Math. Anal. 31 (2000) no. 6, 1192-1213.

[22] J. LigezA, Generalized solutions of ordinary differential equations in the Colombeau algebra, Math. Bohem. 118 (1993), No. 2, 123-146.

[23] J. LigezA, Remarks on generalized solutions of ordinary differential equations in the Colombeau algebra, Math. Bohem. 123 (1998), No. 3, 301-316.

[24] J. E. Marsden, Generalized Hamiltonian mechanics, Arch. Rational Mech. Anal. 28, 323-361 (1968).

[25] P. D. MEтhÉE, Sur les distributions invariantes dans le groupe des rotations de Lorentz, Comment. Math. Helv. 28 (1954), 224-269.

[26] M. Nedelukov, Delta and singular delta locus for one dimensional systems of conservation laws, Preprint, University of Novi Sad (2000).

[27] M. Oberguggenberger, Multiplications of Distributions and Applications to Partial Differential Equations, Longman, 1992.

[28] P. J. Olver, Applications of Lie Groups to Differential Equations, Springer, 2nd ed. New York 1993.

[29] T. Parthasarathy, On Global Univalence Theorems, Lecture Notes in Mathematics 977, Springer 1983.

[30] J. Smoller, Shock Waves and Reaction Diffusion Equations, Springer, New York, 1982.

[31] Z. SZMYDT, On homogeneous rotation invariant distributions and the Laplace operator, Ann. Pol. Math. 6 (1979), 249-259.

[32] Z. Szmydt, Fourier Transformation and Linear Partial Differential Equations, D. Reidel Publ. Comp., Dordrecht 1977.

[33] Z. Szmydt, B. Ziemian, Invariant fundamental solutions of the wave operator, Demonstr. Math. 19, (1986), 371-386.

[34] A. Tengstrand, Distributions invariant under an orthogonal group of arbitrary signature, Math. Scand. 8 (1960), 201-218.

[35] F.Treves, Basic Linear Partial Differential Equations, Academic Press, New York, 1975.

[36] J.A. Vickers, Nonlinear generalised functions in general relativity, in: M. Grosser, G. Hörmann, M. Kunzinger, M. Oberguggenberger (Eds.), Nonlinear Theory of Generalized Functions, 275-290, Chapman \& Hall/CRC, Boca Raton 1999. 